\title{
A Comparative Study Based on Different Control Algorithms for Suppressing Multistage Gear Transmission System Vibrations
}

\author{
Wenhao Sun $\mathbb{D}^{1},{ }^{1}$ Feng Zhang ${ }^{D},{ }^{1}$ Weidong Zhu, ${ }^{2,3}$ Han Wang, \\ Shunan Luo, ${ }^{1}$ and Haiyan $\mathrm{Li}^{1}$ \\ ${ }^{1}$ College of Mechanical Engineering and Automation, Huaqiao University, Xiamen 361021, China \\ ${ }^{2}$ Division of Dynamics and Control, School of Astronautics, Harbin Institute of Technology, Harbin 150001, China \\ ${ }^{3}$ Department of Mechanical Engineering, University of Maryland, Baltimore County, MD 21250, USA \\ Correspondence should be addressed to Wenhao Sun; wilhelmsun@163.com
}

Received 3 November 2017; Revised 8 April 2018; Accepted 19 April 2018; Published 23 May 2018

Academic Editor: Emiliano Mucchi

Copyright (C) 2018 Wenhao Sun et al. This is an open access article distributed under the Creative Commons Attribution License, which permits unrestricted use, distribution, and reproduction in any medium, provided the original work is properly cited.

\begin{abstract}
A modal analysis (MA) was preconsidered to determine a novel active vibration control (AVC) structure of multistage gear transmission system (MGTS) and an appropriate actuating position for the piezoelectric actuator (PZT); the results of the calculating method and the finite element method (FEM) were compared to validate the reliability of MA. The controllers based on different control algorithms were designed to drive the PZTs to output the control force for suppressing the host structure vibrations. To analyze the feasibility of the applied control schemes and discuss the control effects dominated by the different control algorithms, a series of active vibration control numerical simulations were studied. The cosimulation results validate the practicability of the proposed control schemes and provide a forcible guidance for the further experimental works.
\end{abstract}

\section{Introduction}

The presence of the vibrations is a usual problem in mechanical structures, especially in meshing gears. The vibrations of multistage gear transmission system (MGTS) are closely related to the time-varying meshing stiffness, transmission errors, and meshing impacts $[1,2]$. The gear meshing vibrations will be transmitted to the whole housing structure via the transmission shafts and supporting parts, which will damage the properties of the entire transmission system seriously. Over the years, many researchers devoted themselves to solve the critical MGTS active vibration control (AVC) problems. Montague et al. [3] employed an analog phase shifter with an amplifier to drive the piezoelectric actuators (PZT) mounted onto the shaft in the gearbox. The vibration reduced up to $70 \%$ at the fundamental gear mesh frequency (FGMF) in that work. Rebbechi et al. [4] applied two pairs of magnetostrictive actuators fixed on a double row bearing to isolate the vibration transmitting from the supporting structure passing to the housing. An attenuation of $20-28 \mathrm{~dB}$ at FGMF was achieved, but only the first three harmonics were controlled. Chen and Brennan [5] developed a new control scheme that used three magnetostrictive actuators mounted on the gear body. In his research, the actuator according to the control scheme produced circumferential forces to suppress the torsional vibration, and about $7 \mathrm{~dB}$ attenuation was obtained at FGMF of $250 \mathrm{~Hz}$. Guan et al. [6] proposed an active shaft transverse vibration control concept that resides within the gearbox. The system they developed contains a PZT for applying active control force to the shaft via an additive bearing, and an enhanced controller based on filtered-x least mean square (FxLMS) algorithm was designed to generate the actuation control signal. The results show the vibrations reduction up to about $20 \mathrm{~dB}$ at the first two mesh harmonics. Li et al. [7-9] adopted the FxLMS control algorithm to actuate the PZT to produce the active control force for reducing the gearbox vibrations, and the simulation work successfully demonstrated the feasibility of applying the proposed control scheme to gear vibration problems. Based on a combination of feedback and repetitive control strategy, Pinte et al. [10] applied a modular bearing using piezostacks to generate secondary forces for suppressing the noise and 
vibration of the rotation machinery. In addition, Belanger et al. [10-12] developed a finite element model of a Bell 407 transmission and simplified roof structure for investigating the active vibration control problems. An improved LMS control strategy was implemented to reduce the gearbox harmonic tones successfully. Gao et al. [13] studied an active shaft transverse vibration control structure using a modified FxLMS algorithm with online identification to achieve the gearbox vibration suppression, and the simulation results validate the efficiency of the proposed approach. In order to suppress the structure-borne noise and vibration of the rotating machinery, Zhao et al. [14] considered an active vibration control approach using two piezo-based inertial actuators directly mounted on the rotational shaft, and a multi-input-multi-output FxLMS algorithm is employed to generate the appropriate actuation signal. The results show that when the rotational speed is below $180 \mathrm{rpm}$, the plate vibrations reduced more than $7 \mathrm{~dB}$. Recently, Dogruer and Pirsoltan [2] designed a nonlinear controller which can adjust the torque acting on the driving gear to minimize the disadvantageous impact of the time-varying mesh stiffness.

Comparing to the single stage gear transmission system, the vibrations of MGTS aroused by the gear meshing excitations will be more complex. Thus, in the research present here, it is necessary to design a AVC structure of MGTS applying the built-in PZTs. As is known, the positions where the actuator acts on will also have an important influence on the control effect of the whole system. Therefore, confirming a AVC structure of MGTS and an accurate position for the PZT is the primary prerequisite for the following further study. A modal analysis (MA) using calculating method and finite element method (FEM), respectively, was carried out to analyze the natural characteristics of the MGTS. And the AVC structure of MGTS and an appropriate actuating position for the PZT can be determined according to the analysis results of MA. In addition, the Proportional Integral Derivate (PID) algorithm and FxLMS algorithm were applied individually to drive the PZTs to generate control forces for suppressing the complex multi-harmonic vibrations of MGTS. And a more effective control scheme was ascertained by analyzing the control effects of the applied control algorithms in a variety of different conditions. Furthermore, all the practical design parameters were used in the computations to ensure the reliability and convincingness of the cosimulation results. In the next section, the detailed dynamic modelling method and modal parameters solution will be discussed. According to MA results, an appropriate AVC structure will be concluded. Section 3 mainly focuses on the vibration control algorithms, and the keystones of PID and FxLMS control algorithm are illustrated. The cosimulation study for investigating the feasibility of applied control scheme and evaluating the superiority of the two algorithms is carried out in Section 4. At last, the concrete conclusions are discussed after analyzing the relevant cosimulation results.

\section{MA and Structure Description of MGTS}

The dynamic modelling methods of gear transmission system can be mainly divided into two kinds. The first one is the classical modelling methods (CMM), and another one belongs to the modern modelling methods (MMM). The CMM includes two aspects: discretization and mathematicization. In other words, it means a continuous system with infinite DOFs will be discretized into a lumped parameters dynamic model with finite DOFs firstly. Then the dynamic model can be derived into the relevant analytic model by utilizing the mathematicization method. This mentioned discretization method is usually referred to as the lumped parameter method (LPM), which is one of the most commonly used dynamic modelling methods. For instance, $\mathrm{Xu}$ [15], Zhang [16], Ericson [17], and Hammami [18] et al., respectively, used LPM to carry out the dynamic modelling of multistage gear transmission systems. On the other hand, the principle representative of MMM is finite element method (FEM), which can integrate the discretization and mathematicization into a whole. To put it in another way, the FEM can achieve the dynamic modelling process and the derivation process of analytic model combining into one. Therefore, the principle methods discussed here are utilizing LPM to establish a dynamic model of the MGTS to resolve its modal parameters. Furthermore, the FEM will also be applied for carrying out the modal analysis of the MGTS to validate the correctness of both dynamic modelling of the MGTS and modal solution. The concrete contents are given in this section.

2.1. Dynamic Model of MGTS. Considering the torsionalbending vibrations of the transmission shafts and the bending vibrations of the supporting bearings, the equivalent dynamic model of the MGTS with 26 degrees of freedom (DOF) was built as shown in Figure $1 . O_{i} x_{i} y_{i} z_{i}(i=1,2,3,4)$ are the fixed coordinate systems for meshing gears, and $x_{b i} y_{b i} z_{b i}(i=$ $1,2,3,4,5,6)$ are the fixed coordinate systems for the supporting bearings. The figure in the green dashed block diagram on the upper left of Figure 1 denotes the lumped mass model of the meshing gears. $K_{m i}, C_{m i}(i=1,2)$ represent the gear meshing stiffness and damping, respectively, and $e_{i}(i=$ $1,2)$ are the gear meshing error. $K_{b x i}, K_{b y i}, C_{b x i}, C_{b y i}(i=$ $1,2,3,4,5,6)$ denote the radial supporting stiffness and damping of the bearings. $\theta_{i}(i=1,2,3,4)$ represent the angular displacement of the meshing gears. $I_{\text {in }}$ and $I_{\text {out }}$ severally denote the moment of inertia of the motor and load.

In fact, the gear meshing vibrations will change the position of the gear theoretical rotation center, and the dynamic transformation schematic diagram of the gear theoretical center is given in Figure 2(a). The blue solid circles $\left(O_{i} x_{i} y_{i}, i=\right.$ $1,3),\left(O_{j} x_{j} y_{j}, j=2,4\right)$ denote the original position of gears, and the red dashed dot circles $\left(G_{i} x_{i} y_{i}, i=1,3\right),\left(G_{j} x_{j} y_{j}, j=\right.$ $2,4)$ denote the dynamic position of the gear affected by the meshing vibration. More than this, the meshing vibration will also arouse the transmission shafts generating translational and bending deformations. As shown in Figure 2(b), the blue line $B_{1} O_{1} B_{2}$ is the original position of the driving transmission shaft in the steady state condition, and the red dashed line $B_{o 1} G_{1} B_{o 2}$ is the position after deformation owing to the vibrations propagation, projecting $B_{o 1} G_{1} B_{o 2}$ to $x$ - and $y$-axis directions. $B_{o 1 x}, B_{o 1 y}$ and $B_{o 2 x}, B_{o 2 y}$ are the projective coordinate corresponding to $B_{o 1}$ and $B_{o 2}$ in $x$ - and 


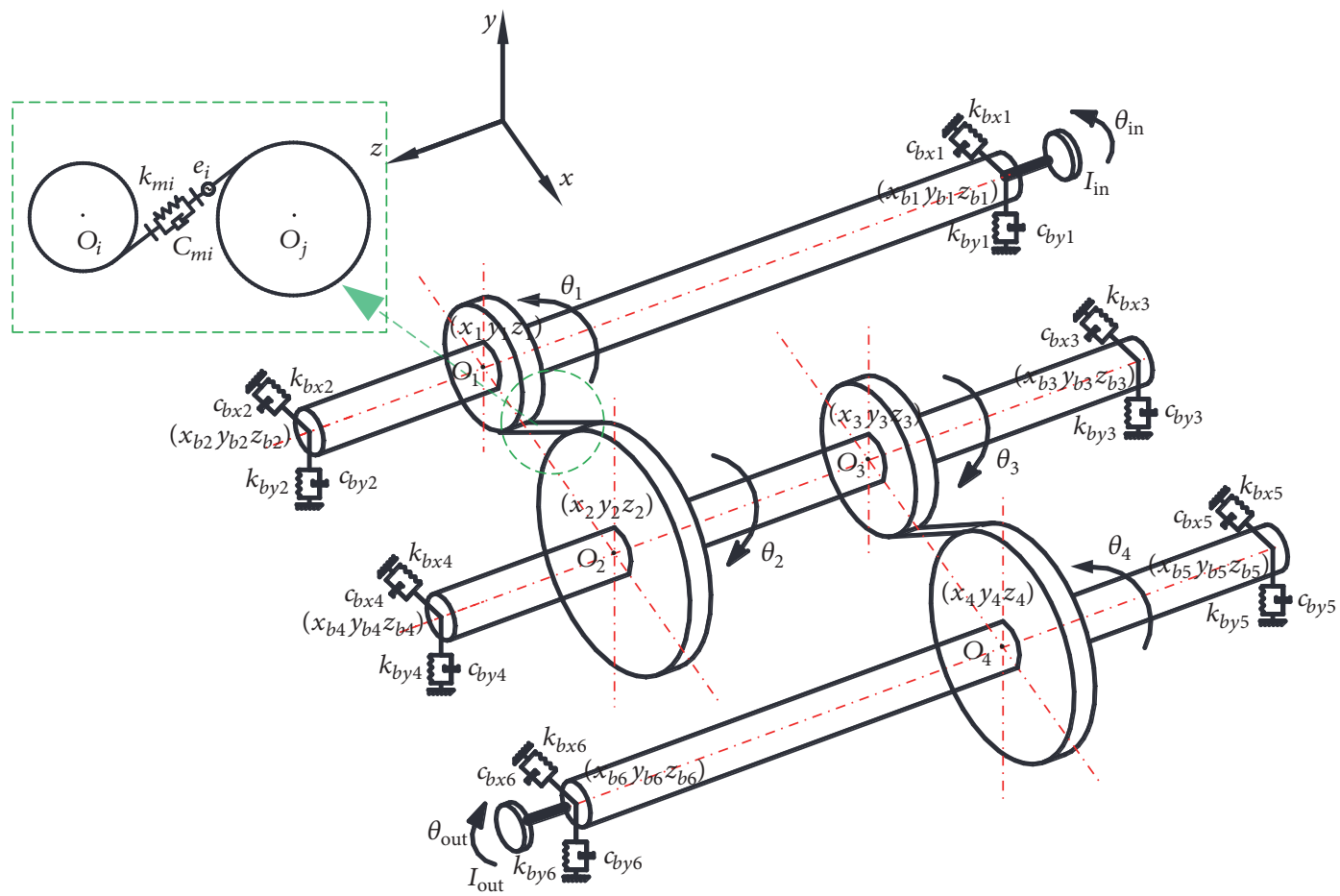

FIGURE 1: The dynamic model of MGTS.

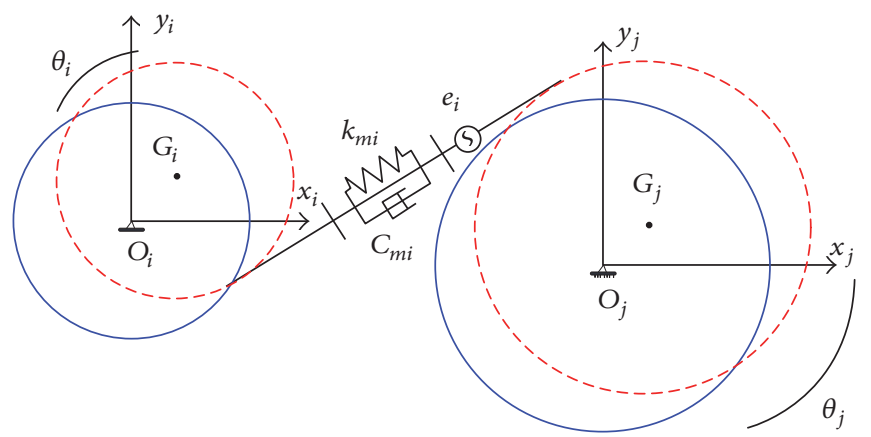

(a)

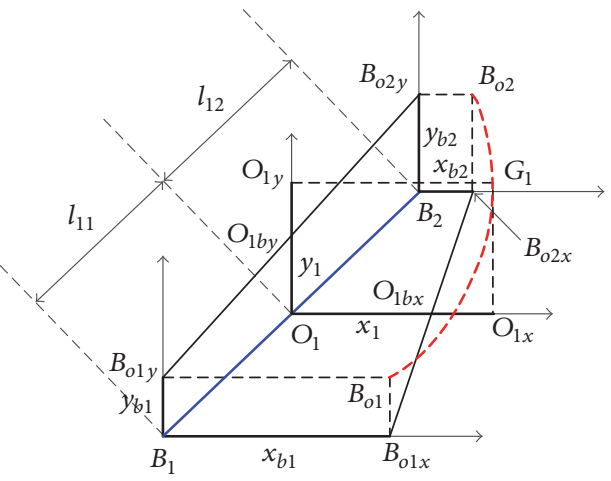

(b)

FIGURE 2: The dynamic transformation schematic diagram of the internal parts of the MGTS.

$y$-axis directions, and $O_{1 x}, O_{1 y}$ is projective coordinate of point $O_{1}$. The distances between the projective points and the coordinate origin can be expressed as follows: $B_{1} B_{o 1 x}=x_{b 1}$, $B_{1} B_{o 1 y}=y_{b 1}, B_{2} B_{o 2 x}=x_{b 2}, B_{2} B_{o 2 y}=y_{b 2}, O_{1} O_{1 x}=x_{1}$, $O_{1} O_{1 y}=y_{1}$. The line segment $B_{o 1 x} B_{o 2 x}$ intersects with line segment $O_{1} O_{1 x}$ at point $O_{1 b x}$. Therefore, the line segment $O_{1} O_{1 b x}$ just denotes the translational deformation without considering the bending deformation. Thus, $O_{1 x} O_{1 b x}$ can be used to represent the bending deformation $\delta_{1}$ of the driving shaft at the location of driving gear rotation center. And $\delta_{1}$ can be divided into $x$ and $y$ direction components $\delta_{1 x}, \delta_{1 y}$. Analogously, the bending deformations in the $x$ and $y$ directions of the transmission shafts at the other meshing gear rotation centers can be expressed as follows:

$$
\delta_{1 x}=x_{1}-\tau_{1} x_{b 2}-\tau_{2} x_{b 1}
$$

$$
\begin{aligned}
& \delta_{1 y}=y_{1}-\tau_{1} y_{b 2}-\tau_{2} y_{b 1} \\
& \delta_{2 x}=x_{2}-\tau_{3} x_{b 4}-\tau_{4} x_{b 3} \\
& \delta_{2 y}=y_{2}-\tau_{3} y_{b 4}-\tau_{4} y_{b 3} \\
& \delta_{3 x}=x_{3}-\tau_{5} x_{b 4}-\tau_{6} x_{b 4} \\
& \delta_{3 y}=y_{3}-\tau_{5} y_{b 4}-\tau_{6} y_{b 3} \\
& \delta_{4 x}=x_{4}-\tau_{7} x_{b 6}-\tau_{8} x_{b 5} \\
& \delta_{4 y}=y_{4}-\tau_{7} y_{b 6}-\tau_{8} y_{b 5}
\end{aligned}
$$

where $\tau_{j}=l_{i j} l_{i}(i=1,2,3,4 ; j=1,2,3,4,5,6,7,8), l_{i j}$ are the distances between the gear rotation centers and the 
bearing theoretical centers, and $l_{i}$ are the lengths between the two bearings theoretical centers located on one shaft. In addition, the deformations $\delta_{m 1}, \delta_{m 2}$ along the meshing lines can be obtained from Figure 2(a):

$$
\begin{aligned}
\delta_{m 1}= & \left(x_{1}-x_{2}\right) \sin \alpha_{1}+\left(y_{1}-y_{2}\right) \cos \alpha_{1}+\theta_{1} r_{1}-\theta_{2} r_{2} \\
& -e_{1} \\
\delta_{m 2}= & \left(x_{3}-x_{4}\right) \sin \alpha_{2}+\left(y_{3}-y_{4}\right) \cos \alpha_{2}+\theta_{3} r_{3}-\theta_{4} r_{4} \\
& -e_{2}
\end{aligned}
$$

where $r_{i}(i=1,2,3,4)$ is the radius of basic circle and $\alpha_{i}(i=$ $1,2)$ are the angle of the working pressure angles.

Assuming $m_{i}(i=1,2,3,4)$ are the lumped mass of the transmitting gears, $m_{b i}(i=1,2,3,4,5,6)$ are the lumped mass of the bearings. $C_{t i}(i=1,2,3)$ and $K_{t i}(i=1,2,3)$ denote the torsional damping and torsional stiffness of the transmission shafts, respectively. $C_{s i}(i=1,2,3,4)$ and $K_{s i}(i=1,2,3,4)$ denote the bending damping and stiffness of the transmission shafts at gear theoretical rotation centers, respectively. And $I_{i}(i=1,2,3,4)$ are the moments of inertial of the transmitting gears, and $T_{\text {in }}, T_{\text {out }}$ denote the torques of the motor and load.

The differential equations of motion (DEOM) can be given by analyzing the relationships among the components of MGTS:

$$
I_{\text {in }} \ddot{\theta}_{\text {in }}+C_{t 1}\left(\dot{\theta}_{\text {in }}-\dot{\theta}_{1}\right)+K_{t 1}\left(\theta_{\text {in }}-\theta_{1}\right)=T_{\text {in }}
$$

Equation (3) is the DEOM of the motor, and the DEOM of the transmitting gears can be expressed from (4) to (7):

$$
\begin{aligned}
& m_{1} \ddot{x}_{1}+C_{s 1} \dot{\delta}_{1 x}+K_{s 1} \delta_{1 x}+C_{m 1} \dot{\delta}_{m 1} \sin \alpha_{1} \\
& \quad+K_{m 1} \delta_{m 1} \sin \alpha_{1}=0 \\
& m_{1} \ddot{y}_{1}+C_{s 1} \dot{\delta}_{1 y}+K_{s 1} \delta_{1 y}+C_{m 1} \dot{\delta}_{m 1} \cos \alpha_{1} \\
& \quad+K_{m 1} \delta_{m 1} \cos \alpha_{1}=0 \\
& I_{1} \ddot{\theta}_{1}+C_{t 1}\left(\dot{\theta}_{1}-\dot{\theta}_{\text {in }}\right)+K_{t 1}\left(\theta_{1}-\theta_{\text {in }}\right)+r_{1} C_{m 1} \dot{\delta}_{m 1} \\
& \quad+r_{1} K_{m 1} \delta_{m 1}=0
\end{aligned}
$$

$$
\begin{aligned}
& m_{2} \ddot{x}_{2}+C_{s 2} \dot{\delta}_{2 x}+K_{s 2} \delta_{2 x}-C_{m 1} \dot{\delta}_{m 1} \sin \alpha_{1} \\
& -K_{m 1} \delta_{m 1} \sin \alpha_{1}=0 \\
& m_{2} \ddot{y}_{2}+C_{s 2} \dot{\delta}_{2 y}+K_{s 2} \delta_{2 y}-C_{m 1} \dot{\delta}_{m 1} \cos \alpha_{1} \\
& -K_{m 1} \delta_{m 1} \cos \alpha_{1}=0 \\
& I_{2} \ddot{\theta}_{2}+C_{t 2}\left(\dot{\theta}_{2}-\dot{\theta}_{3}\right)+K_{t 2}\left(\theta_{2}-\theta_{3}\right)-r_{1} C_{m 1} \dot{\delta}_{m 1} \\
& -r_{2} K_{m 1} \delta_{m 1}=0 \\
& m_{3} \ddot{x}_{3}+C_{s 3} \dot{\delta}_{3 x}+K_{s 3} \delta_{3 x}+C_{m 2} \dot{\delta}_{m 2} \sin \alpha_{2} \\
& +K_{m 2} \delta_{m 2} \sin \alpha_{2}=0 \\
& m_{3} \ddot{y}_{3}+C_{s 3} \dot{\delta}_{3 y}+K_{s 3} \delta_{3 y}+C_{m 2} \dot{\delta}_{m 2} \cos \alpha_{2} \\
& +K_{m 2} \delta_{m 2} \cos \alpha_{2}=0 \\
& I_{3} \ddot{\theta}_{3}+C_{t 2}\left(\dot{\theta}_{3}-\dot{\theta}_{2}\right)+K_{t 2}\left(\theta_{3}-\theta_{2}\right)+r_{3} C_{m 2} \dot{\delta}_{m 2} \\
& +r_{3} K_{m 2} \delta_{m 2}=0 \\
& m_{4} \ddot{x}_{4}+C_{s 4} \dot{\delta}_{4 x}+K_{s 4} \delta_{4 x}-C_{m 2} \dot{\delta}_{m 2} \sin \alpha_{2} \\
& -K_{m 2} \delta_{m 2} \sin \alpha_{2}=0 \\
& m_{4} \ddot{y}_{4}+C_{s 4} \dot{\delta}_{4 y}+K_{s 4} \delta_{4 y}-C_{m 2} \dot{\delta}_{m 2} \cos \alpha_{2} \\
& -K_{m 2} \delta_{m 2} \cos \alpha_{2}=0 \\
& I_{4} \ddot{\theta}_{4}+C_{t 3}\left(\dot{\theta}_{4}-\dot{\theta}_{\text {out }}\right)+K_{t 3}\left(\theta_{4}-\theta_{\text {out }}\right)-r_{4} C_{m 2} \dot{\delta}_{m 2} \\
& -r_{4} K_{m 2} \delta_{m 2}=0
\end{aligned}
$$

The DEOM of the load can be given by

$$
I_{\text {out }} \ddot{\theta}_{\text {out }}+C_{t 3}\left(\dot{\theta}_{\text {out }}-\dot{\theta}_{4}\right)+K_{t 3}\left(\theta_{\text {out }}-\theta_{4}\right)=-T_{\text {out }}
$$

The DEOM of the supporting bearings can be written as

$$
\begin{aligned}
& m_{b i} \ddot{x}_{b i}+C_{b x i} \dot{x}_{b i}+K_{b x i} x_{b i}=F_{b x i} \\
& m_{b i} \ddot{y}_{b i}+C_{b y i} \dot{y}_{b i}+K_{b y i} y_{b i}=F_{b y i}
\end{aligned}
$$

where $C_{b x i}, K_{b x i}(i=1,2,3,4,5,6)$ are the supporting damping and stiffness of the supporting bearings. Because of the deformations of the transmission shafts at the theoretical rotation center of the gears, the supporting reactions of the transmission shaft at the supporting bearings are induced, and it can be expressed as below:

$$
F_{b n i(i=1,2,3,4,5,6)}\left\{\begin{array}{l}
F_{b n 1}=\tau_{2}\left(C_{s 1} \dot{\delta}_{1 m}+K_{s 1} \delta_{1 m}\right) \\
F_{b n 2}=\tau_{1}\left(C_{s 1} \dot{\delta}_{1 m}+K_{s 1} \delta_{1 m}\right) \\
F_{b n 3}=\tau_{4}\left(C_{s 2} \dot{\delta}_{2 m}+K_{s 2} \delta_{2 m}\right)+\tau_{6}\left(C_{s 3} \dot{\delta}_{3 m}+K_{s 3} \delta_{3 m}\right) \\
F_{b n 4}=\tau_{3}\left(C_{s 2} \dot{\delta}_{2 m}+K_{s 2} \delta_{2 m}\right)+\tau_{5}\left(C_{s 3} \dot{\delta}_{3 m}+K_{s 3} \delta_{3 m}\right) \\
F_{b n 5}=\tau_{8}\left(C_{s 4} \dot{\delta}_{4 m}+K_{s 4} \delta_{4 m}\right) \\
F_{b n 6}=\tau_{7}\left(C_{s 4} \dot{\delta}_{4 m}+K_{s 4} \delta_{4 m}\right)
\end{array} \quad(n=x, y)\right.
$$


Equations (1) and (2) are substituted into (3) to (8), and (10) is $=\{\mathbf{P}\}$ substituted into (9), and the equations of motion of the MGTS can be written as

$$
\begin{aligned}
& \mathbf{M}\{\ddot{\mathbf{q}}\}+\left(\mathbf{C}_{\mathbf{b}}+\mathbf{C}_{\mathbf{m}}\right)\{\dot{\mathbf{q}}\}+\left(\mathbf{K}_{\mathbf{b}}+\mathbf{K}_{\mathbf{m}}\right)\{\mathbf{q}\}=\mathbf{T}+\mathbf{F}+\mathbf{E} \\
& \text { The generalized coordinates array is }
\end{aligned}
$$

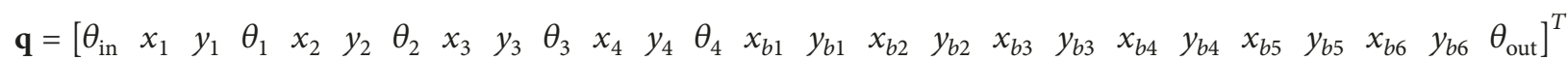

The mass matrix $\mathbf{M}$ can be given as

$$
\mathbf{M}=\operatorname{diag}\left[I_{\text {in }} m_{1} m_{1} I_{1} m_{2} m_{2} I_{2} m_{3} m_{3} I_{3} m_{4} m_{4} I_{4} m_{b 1} m_{b 1} m_{b 2} m_{b 2} m_{b 3} m_{b 3} m_{b 4} m_{b 4} m_{b 5} m_{b 5} m_{b 6} m_{b 6} I_{\text {out }}\right]
$$

The stiffness matrix $\mathbf{K}=\mathbf{K}_{\mathbf{b}}+\mathbf{K}_{\mathbf{m}}$ can be expressed as follows:

$$
K=\left[\begin{array}{ll}
\text { A } & \text { D } \\
B & C
\end{array}\right]
$$

$$
\mathbf{A}=\left[\begin{array}{ll}
\mathbf{A}_{1} & \mathbf{A}_{2} \\
\mathbf{A}_{3} & \mathbf{A}_{4}
\end{array}\right]
$$

where $\mathbf{A}$ is a matrix that consists of four different submatrices $\mathbf{A}_{1}, \mathbf{A}_{2}, \mathbf{A}_{3}, \mathbf{A}_{4}$ :

where $\mathbf{A}_{1}$ is given as follows:

$\mathbf{A}_{1}$

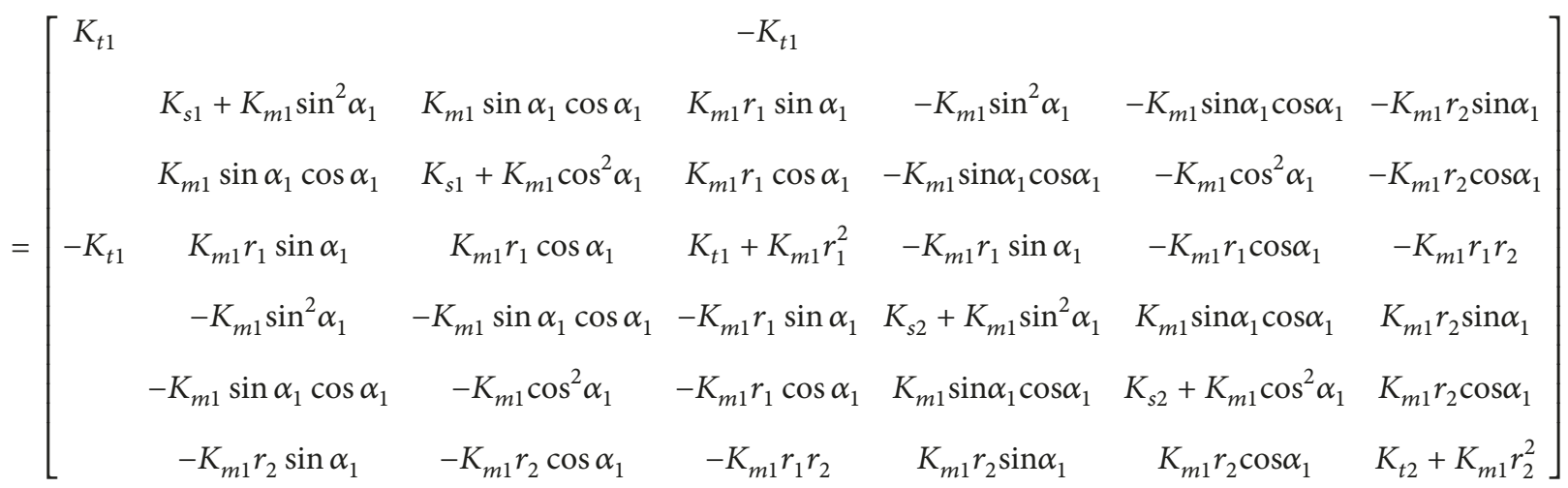

The nonzero element of $\mathbf{A}_{2}$ is $\mathbf{A}_{2(7,3)}=-K_{t 2}$, and the nonzero element of $\mathbf{A}_{3}$ is $\mathbf{A}_{3(3,7)}=-K_{t 2}$, as for $\mathbf{A}_{4}$, it can be expressed as below:

$$
\mathbf{A}_{4}=\left[\begin{array}{cccccc}
K_{s 3}+K_{m 2} \sin ^{2} \alpha_{2} & K_{m 2} \sin \alpha_{2} \cos \alpha_{2} & K_{m 2} r_{3} \sin \alpha_{2} & -K_{m 2} \sin ^{2} \alpha_{2} & -K_{m 2} \sin \alpha_{2} \cos \alpha_{2} & -K_{m 2} r_{4} \sin \alpha_{2} \\
K_{m 2} \sin \alpha_{2} \cos \alpha_{2} & K_{s 3}+K_{m 2} \cos ^{2} \alpha_{2} & K_{m 2} r_{3} \cos \alpha_{2} & -K_{m 2} \sin \alpha_{2} \cos \alpha_{2} & -K_{m 2} \cos ^{2} \alpha_{2} & -K_{m 2} r_{4} \cos \alpha_{2} \\
K_{m 2} r_{3} \sin \alpha_{2} & K_{m 2} r_{3} \cos \alpha_{2} & K_{t 2}+K_{m 2} r_{3}^{2} & -K_{m 2} r_{3} \sin \alpha_{2} & -K_{m 2} r_{3} \cos \alpha_{2} & -K_{m 2} r_{3} r_{4} \\
-K_{m 2} \sin ^{2} \alpha_{2} & -K_{m 2} \sin \alpha_{2} \cos \alpha_{2} & -K_{m 2} r_{3} \sin \alpha_{2} & K_{s 4}+K_{m 2} \sin ^{2} \alpha_{2} & K_{m 2} \sin \alpha_{2} \cos \alpha_{2} & K_{m 2} r_{4} \sin \alpha_{2} \\
-K_{m 2} \sin \alpha_{2} \cos \alpha_{2} & -K_{m 2} \cos ^{2} \alpha_{2} & -K_{m 2} r_{3} \cos \alpha_{2} & K_{m 2} \sin \alpha_{2} \cos \alpha_{2} & K_{s 4}+K_{m 2} \cos ^{2} \alpha_{2} & K_{m 2} r_{4} \cos \alpha_{2} \\
-K_{m 2} r_{4} \sin \alpha_{2} & -K_{m 2} r_{4} \cos \alpha_{2} & -K_{m 2} r_{3} r_{4} & K_{m 2} r_{4} \sin \alpha_{2} & K_{m 2} r_{4} \cos \alpha_{2} & K_{t 3}+K_{m 2} r_{4}^{2}
\end{array}\right]
$$


The nonzero element of $\mathbf{B}$ is $\mathbf{B}_{(13,13)}=-K_{t 3}$, and the matrix $\mathrm{C}$ can be given as follows:

$$
\mathrm{C}=\operatorname{diag}\left[K_{b x 1} K_{b y 1} K_{b x 2} K_{b y 2} K_{b x 3} K_{b y 3} K_{b x 4} K_{b y 4} K_{b x 5} K_{b y 5} K_{b x 6} K_{b y 6} K_{t 3}\right]
$$

The matrix $\mathbf{D}$ is expressed as below:

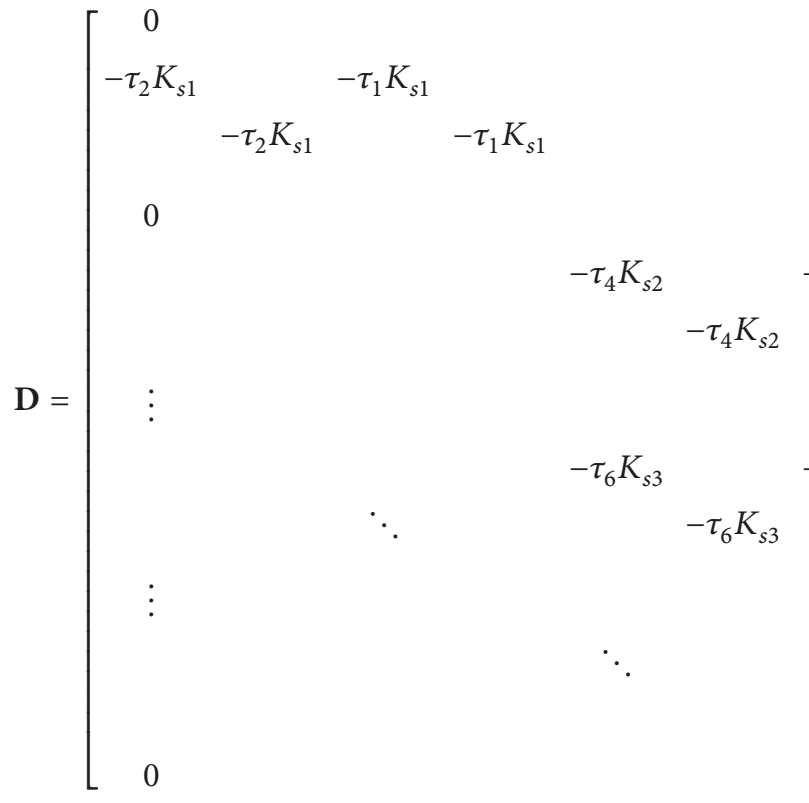

The damping matrix $\mathbf{C}^{\prime}=\mathbf{C}_{\mathbf{b}}+\mathbf{C}_{\mathbf{m}}$ can be obtained by replacing the stiffness parameters with corresponding damping parameters. The external load matrix is given in (22).

2.2. Modal Analysis of MGTS Based on FEM. In this paper, determining an appropriate AVC structure of the MGTS is a primary task for reducing the vibration magnitude of the MGTS, and ascertaining an accurate actuating position for the PZT is a key objective. Furthermore, another noteworthy problem is the excitation frequencies that should be selected carefully to avoid the entire system generating resonances. Therefore, a MA was carried out to solve the above problems.

For the undamped free vibration system, the vibration equations can be obtained by simplifying (11), as shown in (20):

$$
M \ddot{q}+K q=0
$$

The essence of modal solving process is a method for decoupling the differential equations, converting the physical coordinates of the differential equations to modal coordinates. Thus, (17) can be written as follows:

$$
\left(K-w_{i}^{2} M\right) \varphi_{i}=0
$$

$$
\mathbf{P}=\left[\begin{array}{c}
T_{\text {in }} \\
C_{m 1} \dot{e}_{1} \sin \alpha_{1}+K_{m 1} e_{1} \sin \alpha_{1} \\
C_{m 1} \dot{e}_{1} \cos \alpha_{1}+K_{m 1} e_{1} \cos \alpha_{1} \\
r_{1} C_{m 1} \dot{e}_{1}+r_{1} K_{m 1} e_{1} \\
-C_{m 1} \dot{e}_{1} \sin \alpha_{1}-K_{m 1} e_{1} \sin \alpha_{1} \\
-C_{m 1} \dot{e}_{1} \cos \alpha_{1}-K_{m 1} e_{1} \cos \alpha_{1} \\
-r_{2} C_{m 1} \dot{e}_{1}-r_{2} K_{m 1} e_{1} \\
C_{m 2} \dot{e}_{2} \sin \alpha_{2}+K_{m 2} e_{2} \sin \alpha_{2} \\
C_{m 2} \dot{e}_{2} \cos \alpha_{2}+K_{m 2} e_{2} \cos \alpha_{2} \\
r_{3} C_{m 2} \dot{e}_{2}+r_{3} K_{m 2} e_{2} \\
-C_{m 2} \dot{e}_{2} \sin \alpha_{2}-K_{m 2} e_{2} \sin \alpha_{2} \\
-C_{m 2} \dot{e}_{2} \cos \alpha_{2}-K_{m 2} e_{2} \cos \alpha_{2} \\
-r_{4} C_{m 2} \dot{e}_{2}-r_{4} K_{m 2} e_{2} \\
0 \\
\vdots \\
0 \\
-T_{\mathrm{out}}
\end{array}\right]
$$


TABLE 1: Parameters of MGTS.

\begin{tabular}{lcc}
\hline Variable & Numerical value & Unit \\
\hline$m_{1} / m_{2}$ & $0.53 / 1.264$ & $\mathrm{Kg}$ \\
$m_{3} / m_{4}$ & $0.56 / 1.526$ & $\mathrm{Kg}$ \\
$m_{b i}$ & 0.25 & $\mathrm{Kg}$ \\
$r_{1} / r_{2}$ & $0.19 / 0.37$ & $\mathrm{M}$ \\
$r_{3} / r_{4}$ & $0.23 / 0.35$ & $\mathrm{M}$ \\
$\alpha_{1} / \alpha_{2}$ & $20 / 20$ & $\left({ }^{\circ}\right)$ \\
$K_{t 1}$ & $8.0024 \times 10^{4}$ & $\mathrm{~N} / \mathrm{m}$ \\
$K_{t 2}$ & $8.0425 \times 10^{4}$ & $\mathrm{~N} / \mathrm{m}$ \\
$K_{t 3}$ & $5.056 \times 10^{4}$ & $\mathrm{~N} / \mathrm{m}$ \\
$K_{s 1}$ & $2.4972 \times 10^{7}$ & $\mathrm{~N} / \mathrm{m}$ \\
$K_{s 2}$ & $2.3162 \times 10^{7}$ & $\mathrm{~N} / \mathrm{m}$ \\
$K_{s 3}$ & $2.7134 \times 10^{7}$ & $\mathrm{~N} / \mathrm{m}$ \\
$K_{s 4}$ & $1.9721 \times 10^{7}$ & $\mathrm{~N} / \mathrm{m}$ \\
$K_{b}$ & $5.5 \times 10^{7}$ & $\mathrm{~N} / \mathrm{m}$ \\
$I_{\text {in }}$ & 0.0614 & $\mathrm{~kg} \cdot \mathrm{m}^{2}$ \\
$I_{1}$ & $9.416 \times 10^{-4}$ & $\mathrm{~kg} \cdot \mathrm{m}^{2}$ \\
$I_{2}$ & $8.05 \times 10^{-4}$ & $\mathrm{~kg} \cdot \mathrm{m}^{2}$ \\
$I_{3}$ & $9.042 \times 10^{-4}$ & $\mathrm{~kg} \cdot \mathrm{m}^{2}$ \\
$I_{4}$ & $9.416 \times 10^{-4}$ & $\mathrm{~kg} \cdot \mathrm{m}^{2}$ \\
$I_{\text {out }}$ & 0.0144 & $\mathrm{~kg} \cdot \mathrm{m}^{2}$ \\
Poisson ratio & 0.3 & $\mathrm{Kg} / \mathrm{m}^{3}$ \\
Density & $7.85 \times 10^{3}$ & $\mathrm{MPa}$ \\
Young's Modulus & $2 \times 10^{5}$ & \\
\hline
\end{tabular}

In (22), $w_{i}, \varphi_{i}(i=1,2, \ldots, n)$ denote the $i$ th order natural frequency and the $i$ th order modal shape of the MGTS. For the free vibration system, lower order vibration modals have more prominent contribution rate and determine the dynamic response characteristics of the system [19], and forasmuch, the 1st- to 6th-order modal parameters are extracted by calculation method and FEM, respectively. And the main parameters for calculating the modal parameters of the MGTS are given in Table 1.

As for the modal parameters solved by FEM, firstly, the three-dimensional model of MGTS was built in the UG NX software.

Then the model was exported as a Parasolid file into ANSYS Workbench platform. Mechanic properties of materials used for the modal analysis are elastic modulus, Poisson's ratio, and material density; these parameters are given in Table 1. Using automatic mesh generation, then the preceding six-order modal parameter was extracted. The threedimension model and the meshed model of MGTS are given in Figures 3(a) and 3(b), respectively. And the comparative analysis of the natural frequencies with the two methods is given in Table 2.

From Table 2, we can find that the general relative error rate between the two solutions is less than $5 \%$, which is theoretically permissible. To some extent, the dynamic model of MGTS established in forward has been validated. Furthermore, the preceding six-order mode shapes are arrayed in proper sequence as shown in Figures 4(a)-4(f). From Figure 4, we can find that the 1st-, 4th-, and 6th-order modal
TABLE 2: The comparisons of natural frequency by two different methods.

\begin{tabular}{lccc}
\hline Order & Calculation method & FEM & Error rate \\
\hline 1st & 1547.2 & 1587 & $2.5 \%$ \\
2nd & 1889.82 & 1938.2 & $2.49 \%$ \\
3rd & 2261.2 & 2328.3 & $2.88 \%$ \\
4th & 2588.4 & 2656.5 & $2.56 \%$ \\
5th & 3268.94 & 3288 & $0.57 \%$ \\
6th & 3299.51 & 3324.3 & $0.74 \%$ \\
\hline
\end{tabular}

shapes are mainly taking on the bending vibration, and the 2nd-, 3rd-, and 5th-order modal shapes are mainly taking on bending-torsional coupling vibration. In general, the system will generate some modal vibrations due to the influence of the work load. And the total system response at work is a linear superposition of the response of each excited mode. Therefore, it is necessary to analyze the modals of MGTS for determining a AVC structure and a position where the PZTs should act on. According to Figure 4, it is apparent to know that the max deformation of the gears mainly appeared at gear teeth and its inside. And it is too hard to fix the PZTs on the gear teeth firmly during work. However, the max and main deformation for the transmission shafts mainly appeared at the neighbourhood of the gears; thus it is a good choice to apply the PZT working on the transmission shafts. Therefore, this paper applied a pair of PZTs acting on the high speed and low speed shafts. This active shaft control concept can be verified by a series of cosimulations; furthermore, a better actuating position for the PZTs can also be obtained by the numerical simulations, all of these will be discussed in detail in Section 4. And the details of the MGTS are discussed in the next section.

2.3. Structure Description of MGTS. The mesh excitations interact with the MGTS dynamic characteristics to generate the time-varying mesh forces, which will bring about the complex vibrations. Then the vibration energy is transmitted through the transmission shafts and supporting parts to the host housing, but just because the existence and transmitting of the vibration will have a severe effect on the working performance of the MGTS. Hence, a AVC structure for reducing the MGTS vibrations was developed based on the previous MA in this paper. The three-dimensional view of the MGTS and the cross-sectional view expounding the inner details of the proposed outfit are shown in Figures 5(a) and 5(b). The main structural configuration includes a motor, a load, a pair of PZTs, and the MGTS. The motor and load are connected with MGTS through a pair of couplings. As for the PZTs, they can be mounted on the auxiliary supports, and the auxiliary supports can be mounted on both side walls of the gearbox housing.

Furthermore, because the transmission shafts may generate vibration deformation, the PZTs may have some lateral motions that may affect the PZTs. Hence, the setup must be carefully designed such that the PZTs should not be directly connected to the additional bearing raceway. Just because of this, a decompression unit is immobilized between the PZT 


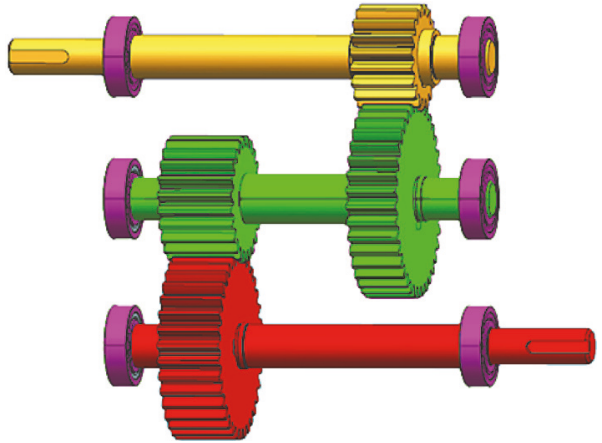

(a)

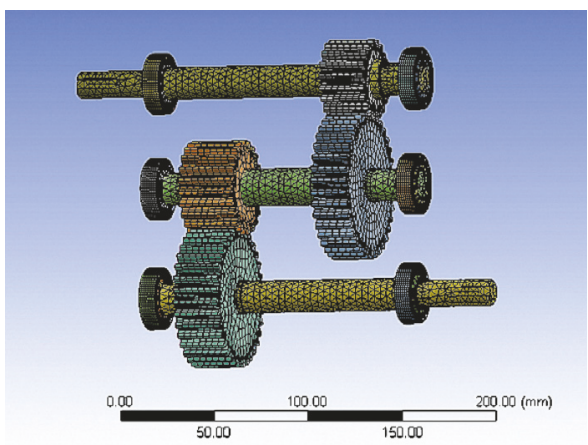

(b)

FIGURE 3: The meshed modal of MGTS.

and the addition bearing for cutting down the longitudinal force to avoid PZT generating unnecessary damage, and the detailed view is given in Figure 5(a).

\section{Vibration Control Algorithms}

The vibration controller, as the most critical part of the overall vibration control system, has a significant effect on the final control results. Therefore, the control algorithm, as the core of the controller, should be carefully designed for obtaining better control effects. However, most of the traditional control means need an accurate controlled model. But, as for the MGTS, it is pretty hard to build a precise model because its parameters are uncertain. Furthermore, the response characteristic of MGTS is time-variant; therefore, it is not easy to design a constant control system to attain the anticipated requirements using the classical control methods. In this paper, for the purpose of suppressing the vibration of the MGTS, the PID control method and FxLMS adaptive control algorithm were used to establish corresponding control schemes, respectively. The details of these algorithms will be discussed as follows.

3.1. PID Control Algorithm. The PID control strategy is the most popular feedback control method used in various industrial controls due to its simple structure, easy implementation, good stability, and strong robustness. As for the classical control method, it is commendable that it has been successfully used for over 50 years. Thus far, PID control is still the basic control method in the process industries. PID control technology and its optimized control technology are accounted for more than $90 \%$ in accordance with the survey data $[20,21]$. The PID control algorithm mainly includes the proportional (P), integral (I), and derivative (D) three modes. The three modes are also the dominant factor for determining the control effect. The system stability mainly depends on adjusting the $\mathrm{P}$ control gain parameter. The steady state error can be controlled by adjusting the I control gain parameter. And the D control has an important effect on increasing the stability of the system and suppressing the overshoot. Thus, determining the three parameters of $\mathrm{P}, \mathrm{I}$, and $\mathrm{D}$ is the main mission for designing the PID controller [22]. The block
TABLE 3: Numerical process of FxLMS algorithm.

\begin{tabular}{lc}
\hline Description & Algorithm \\
\hline Initialization & $\omega(0)=0$ \\
Update & $y(n)=\omega(n) * r(n)$ \\
& $r^{\prime}(n)=S^{\prime}(n) * r(n)$ \\
(iteration) & $e(n)=d(n)-y(n) * S(n)$ \\
& $\omega(n+1)=\omega(n)+\mu r^{\prime}(n) e(n)$ \\
\hline
\end{tabular}

diagram of PID control is given in Figure 6(a), and the output of PID controller in time domain is given as below:

$$
\begin{aligned}
& u(t)=K_{p} e(t)+K_{i} \int_{t_{1}}^{t_{2}} e(\tau) d \tau+K_{d} \frac{d e(t)}{d t} \\
& e(t)=x(t)-y(t)
\end{aligned}
$$

where $e(t)$ is the error signal, $u(t)$ is the output of the controller, and $K_{p}, K_{i}$, and $K_{d}$ represent the $\mathrm{P}$ gain, I gain, and $\mathrm{D}$ gain, respectively.

3.2. FxLMS Control Algorithm. The FxLMS algorithm is one of the most popular algorithms which has been employed in many other active vibration control studies [23-26]. The FxLMS algorithm control law is utilized for the sake of achieving the high-performance control effect. It does not rely on the prior knowledge and the specific model; moreover, it shows insensitivity when facing the variation of the model parameters. As a matter of fact, the FxLMS algorithm is one of the most effective methods based on the standard LMS algorithm. The existence of the secondary path which is known as one path between the output of adaptive controller and the housing response point may cause the traditional LMS algorithm used for AVC applications to be unstable. As a consequence, a secondary path estimation model is used as a compensator to address this problem. The control schematic diagram of FxLMS algorithm is given in Figure 6(b), and the numerical process of this algorithm is given in Table 3.

The structure of FxLMS algorithm is shown in Figure $6(\mathrm{~b}) . H(z)$ denotes the transfer function between the error excitation signal $x(n)$ and the undealt gear housing vibration $d(n) . S(z)$ represents the secondary path transfer 


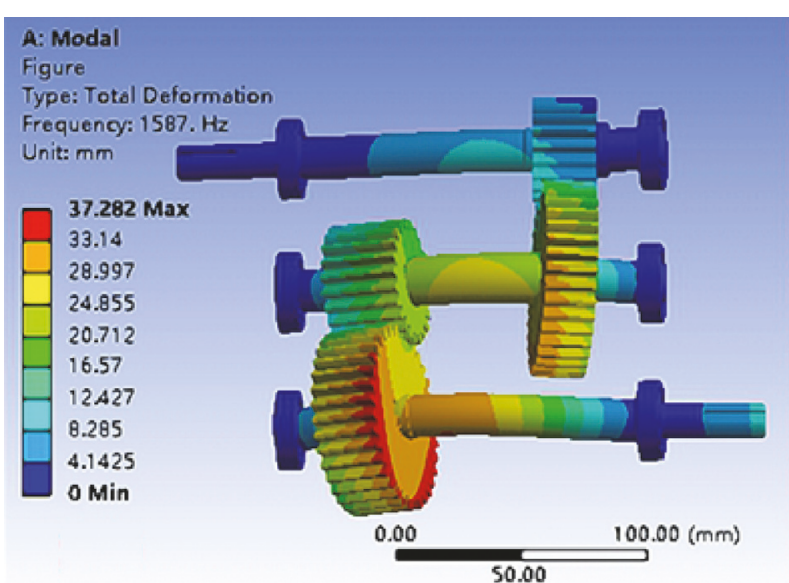

(a)

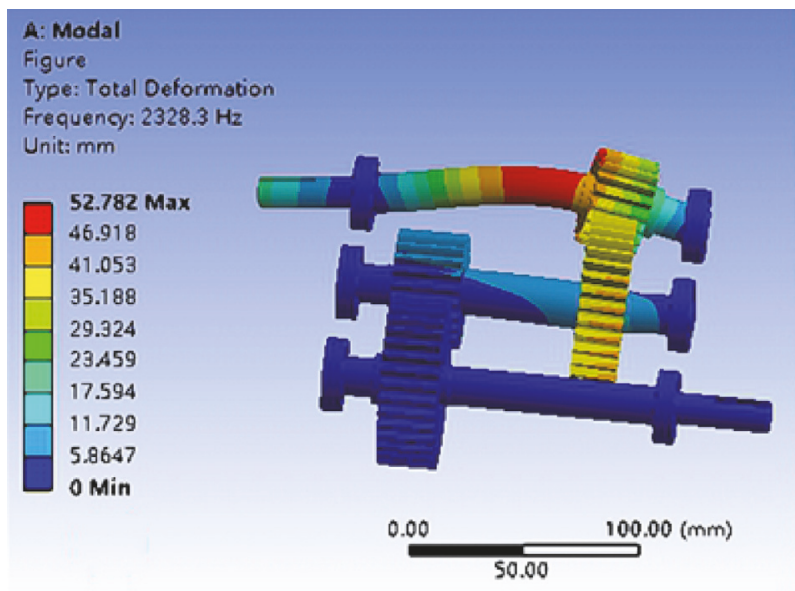

(c)

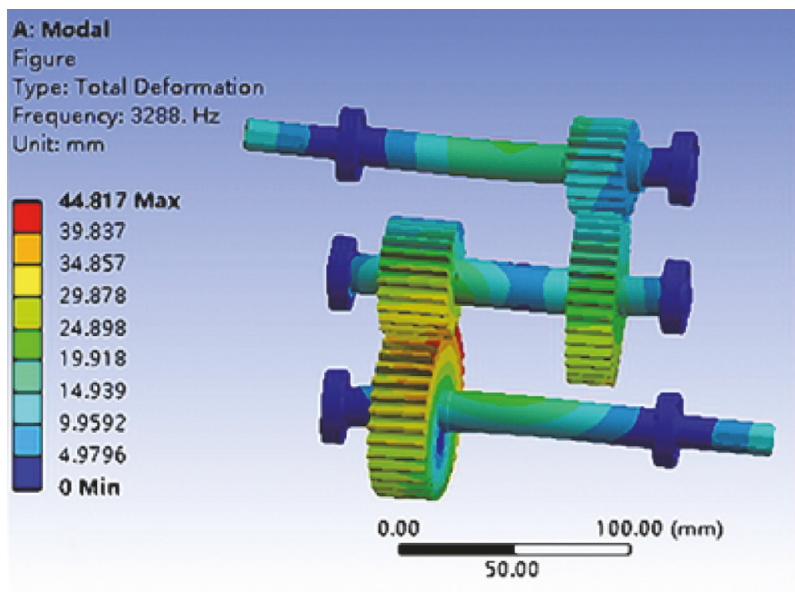

(e)

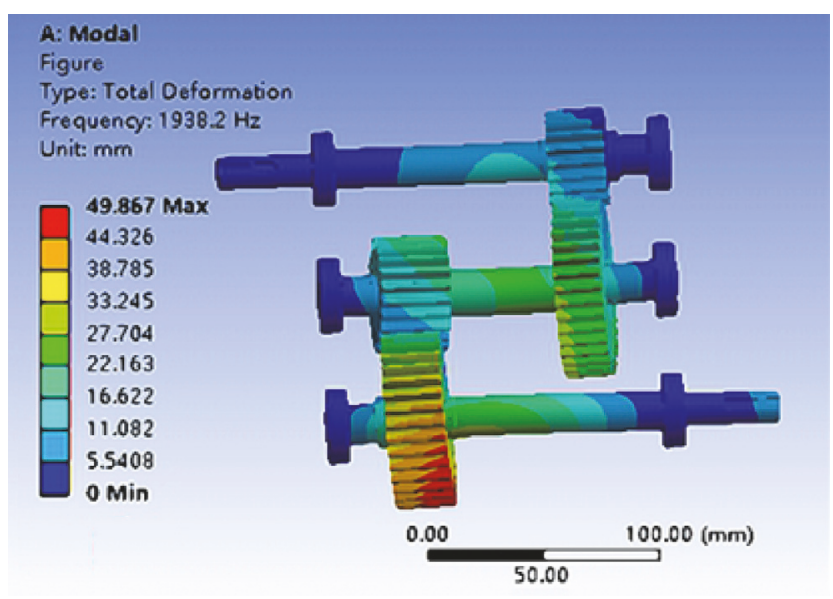

(b)

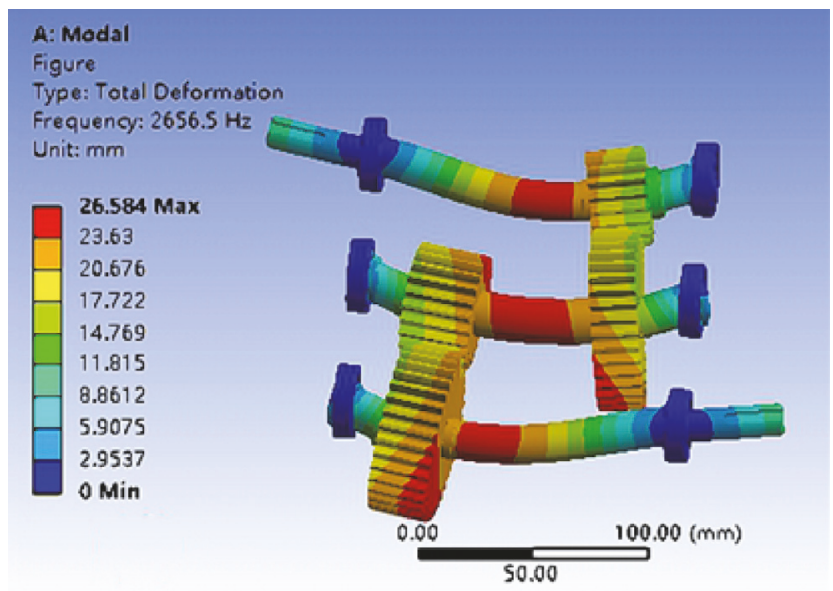

(d)

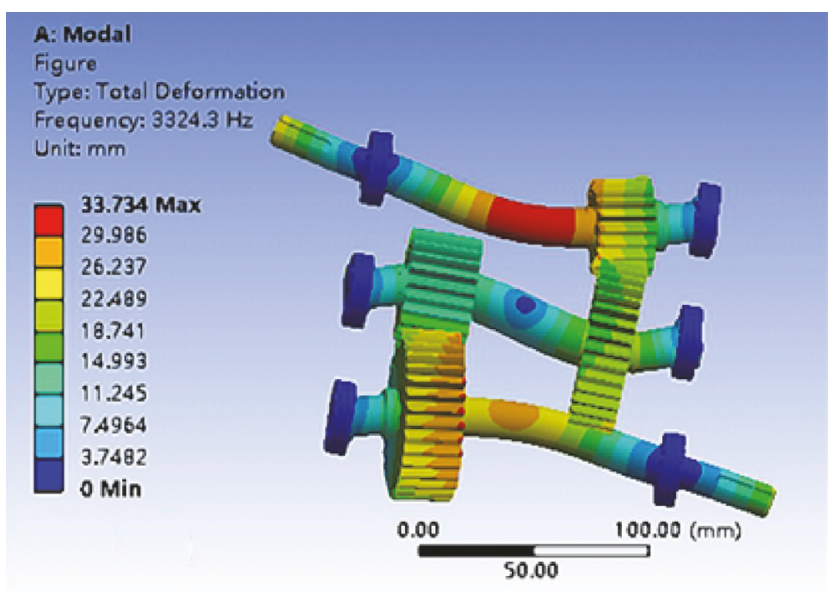

(f)

FIGURE 4: The preceding six-order modal shapes of MGTS.

function between the output terminal of the controller and the gearbox housing vibration. $S^{\prime}(z)$ is the estimated model of $S(z)$. The error signal $e(n)$ is equal to acceleration along $y$ direction, the housing vibration response along the gear line-of-action which can be measured through an accelerometer. Moreover, $e(n)$ participates in the FxLMS algorithm iteration for adjusting the adaptive controller's weight $\omega(z)$, $\mu$ is the iteration step, and $y(n)$ is the controller output signal. 


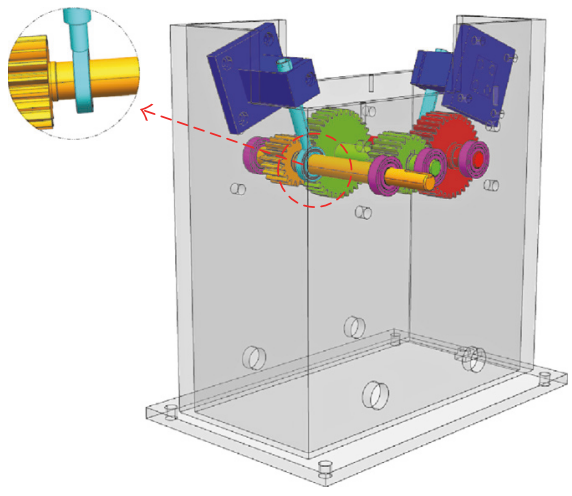

(a)

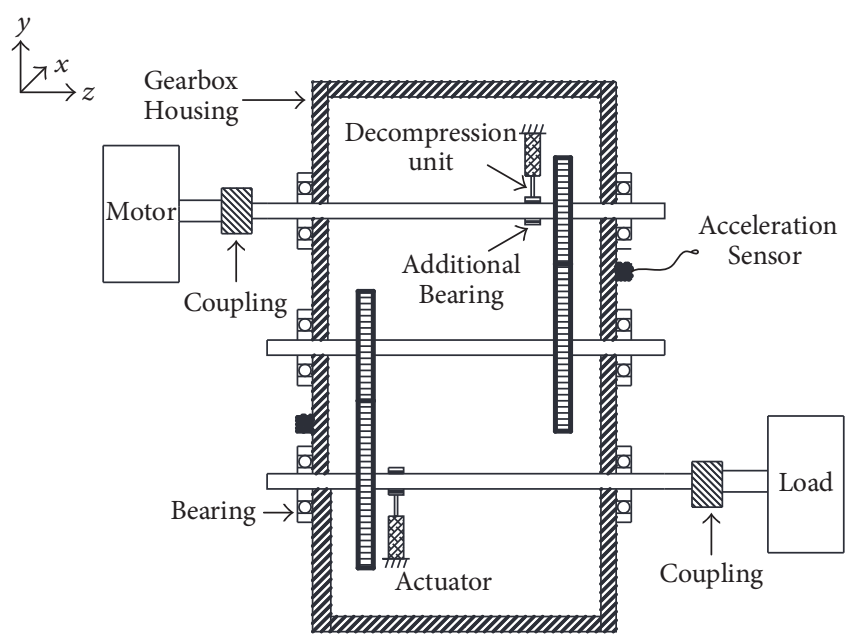

(b)

FIgURE 5: The three-dimension and cross-sectional view of MGTS.

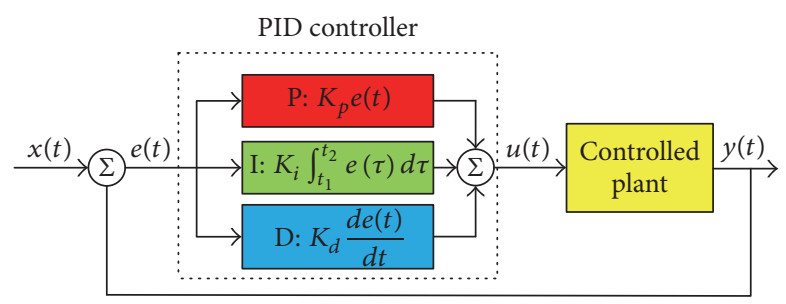

(a)

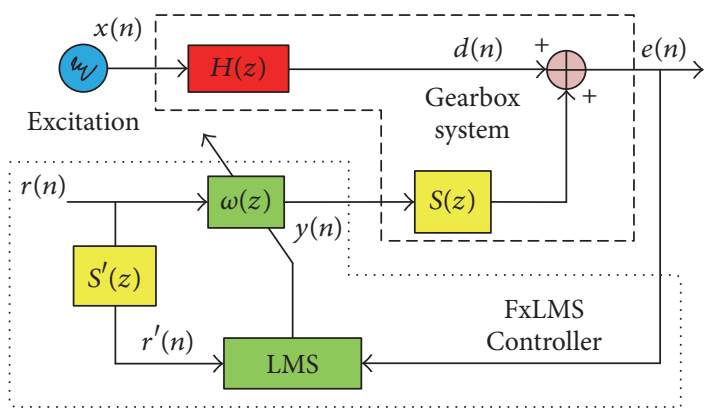

(b)

FIGURE 6: The schematic diagram of PID control algorithm and FxLMS algorithm.

\section{Numerical Simulations}

Although the corresponding dynamic analysis can be carried out through the dynamic model established by using LPM, the LPM cannot meet the requirements when it comes to the structural analysis, especially the problem of PZT placement optimization which will be discussed in detail in this paper. Furthermore, the virtual prototype established in ADAMS is more accurate and comprehensive, which can reflect the interior relevant relationships of the components of the MGTS by adding the corresponding constraint relation, such as the spherical constraint, revolute joint, and specific contact constraint, while the lumped parameter model cannot achieve this key point effectively. On the other hand, the cosimulation technique proposed here is suitable for the testing of mechanical structure design and analyzing a whole system behavior and testing of the control system. Therefore, the cosimulations based on Matlab/Simulink and ADAMS will be carried out for testing the feasibility of the AVC scheme adopted in this paper and discussing the control effects dominated by different control algorithms. And the superiority of the two control algorithms used here will be evaluated as well.
In view of the analytic results of the above two sections, the AVC analysis of MGTS based on PID and FxLMS algorithms is considered in this section. The diagrammatic sketch of the MGTS with controlled system is given in Figure 7.

In Figure 7, the No. 1 acceleration sensor is used for acquiring the reference signal for the control algorithm and the No. 2 acceleration sensor is used for picking up the error signal that needs to feed back to the control algorithm. Then the controller outputs the control command to drive the PZTs generating active control force to suppress the vibrations of MGTS. Therefore, to ensure that the analytic results can reflect the realistic situation enough, a cosimulation technique is utilized by two well-known software programmes, ADAMS and Matlab/Simulink. The ADAMS and Matlab/Simulink cosimulation can be mainly divided into two parts. In terms of the first part, first of all, the virtual prototype model of MGTS is established in ADAMS. And the vital mission of this project is to determine the appropriate constraints according to the relationship between all components of the MGTS to guarantee the virtual prototype model up to the actual criterion as far as possible. As for the second important part, the FxLMS adaptive control algorithm is performed by using the user-defined level-2 $S$ function in 


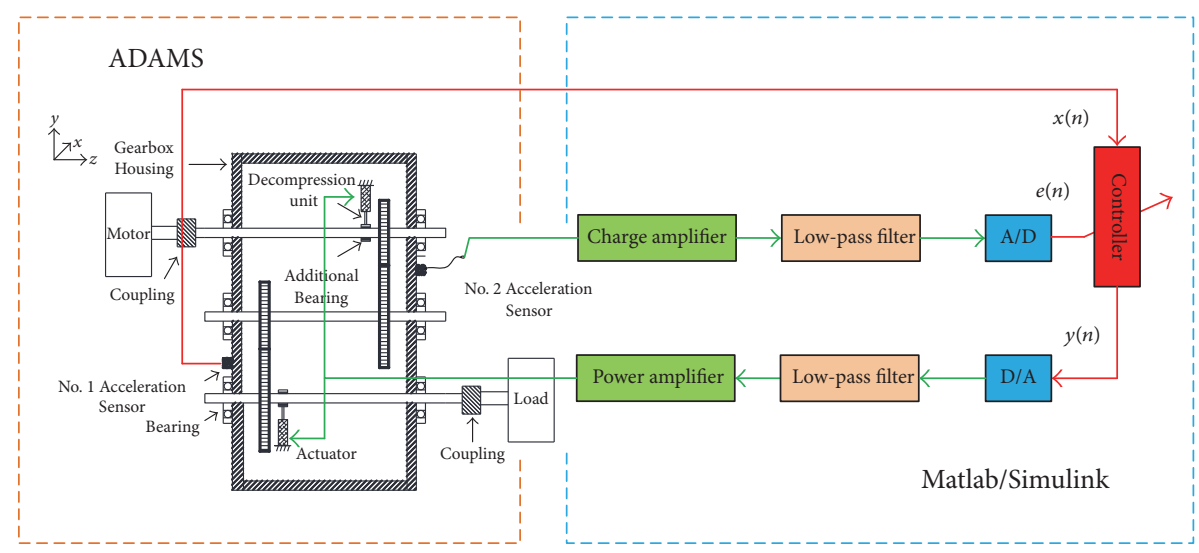

FIGURE 7: The diagrammatic sketch of the MGTS with controlled system.

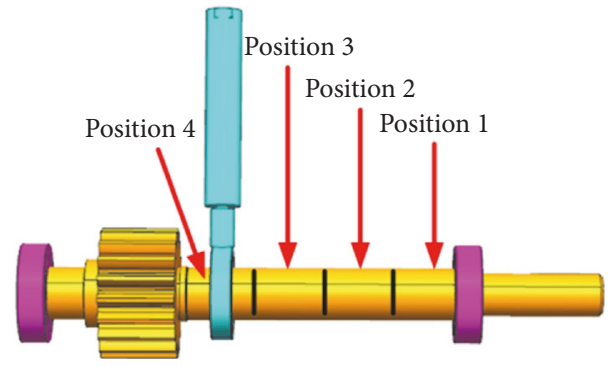

(a)

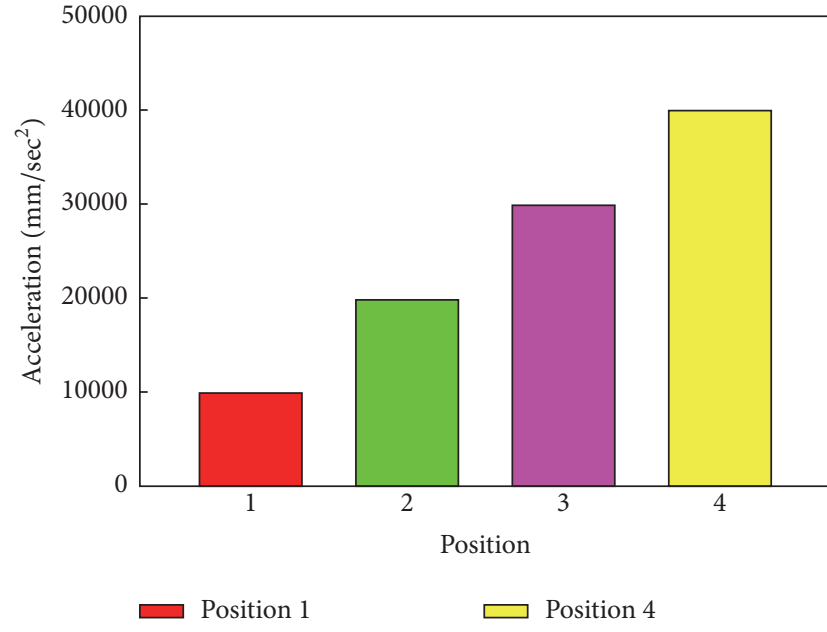

(b)

FIGURE 8: The diagrammatic sketch of the actuating position for PZTs and the vibration magnitudes at the corresponding position.

Matlab. Then the cosimulation control structure based on PID and FxLMS algorithm is established in Simulink.

To validate the effectiveness of the proposed method above, the connection between ADAMS and Matlab is established by importing the virtual prototype into Matlab to achieve the interactive simulations. The cosimulation analysis is carried out under the circumstance of the rotational speed and load under steady state condition. That means both of the magnitudes of rotational speed and load remain constant, and the MGTS works under steady state conditions. As discussed by Li et al. [7], for the FGMF, it has been proved that the proposed control method did not perform well and can not achieve significant control at this frequency. Thus, the following study will mainly focus on the single target harmonic vibration control.

4.1. PZT Placement Optimization. For testifying the proposed active shaft control concept according to the results of the modal analysis discussed in Section 2 and finding an appropriate actuating position for the PZT, a series of cosimulations were carried out. The input shaft was taken as an example for analyzing the possibility of the active shaft control concept. It is clear that the position on the gear's left is not fit to locate one PZT. Therefore the input shaft is roughly divided into four parts for locating the PZTs, positions 1-4, as shown in Figure 8(a). According to the generation principle of the gear mesh vibrations, the more closer the position to the gear, the greater the amplitude. Thus, the vibration magnitudes for the corresponding positions are given in Figure 8(b). We set the input rotational speed $n=1524 \mathrm{r} / \mathrm{min}$ and the load torque $T=1 \mathrm{~N} \cdot \mathrm{m}$. With regard to the four different positions shown in Figure 8(a), the corresponding cosimulation analysis is performed under the same condition. The control variate method can avoid the influences of the other complex factors and ensure the accuracy of the cosimulation results. The cosimulation results are given in Figure 9. 

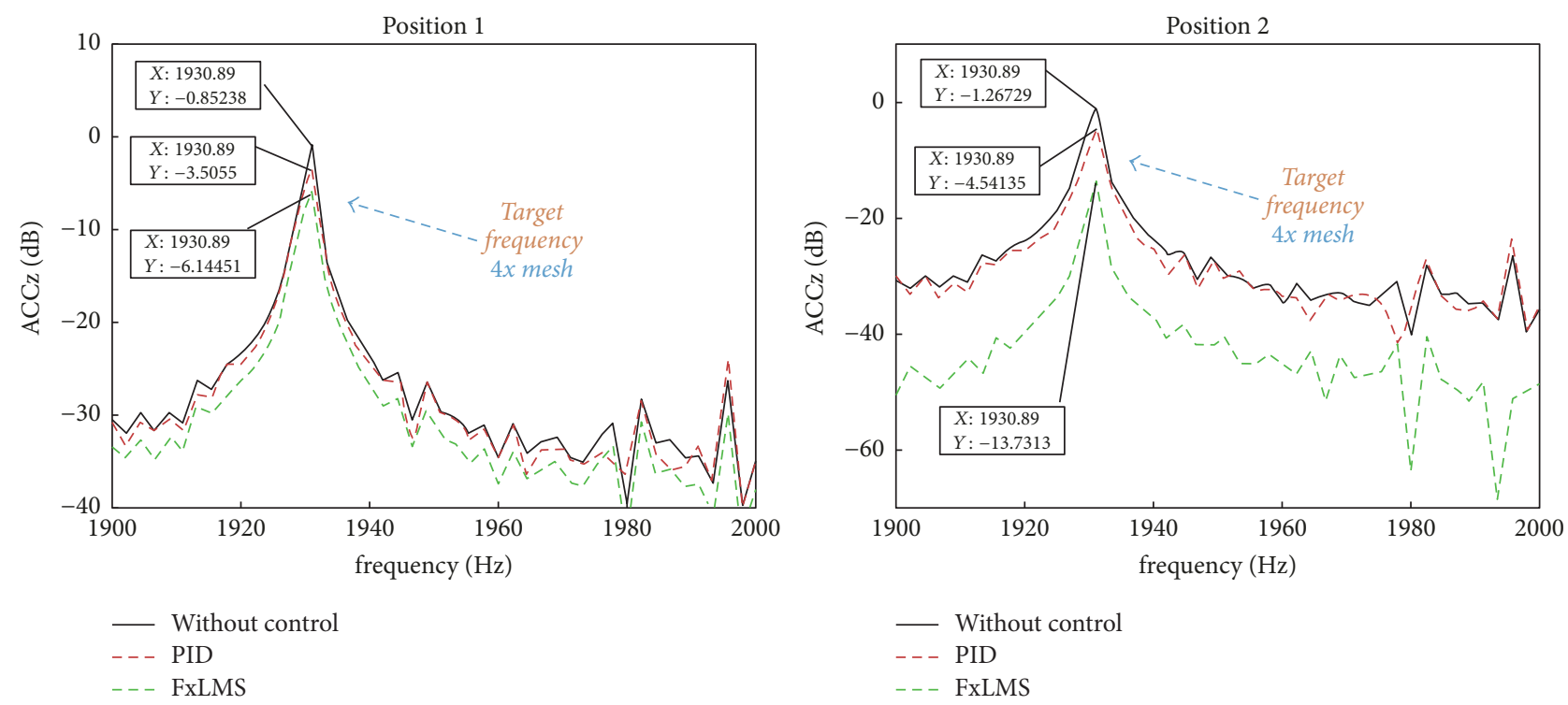

Position 3
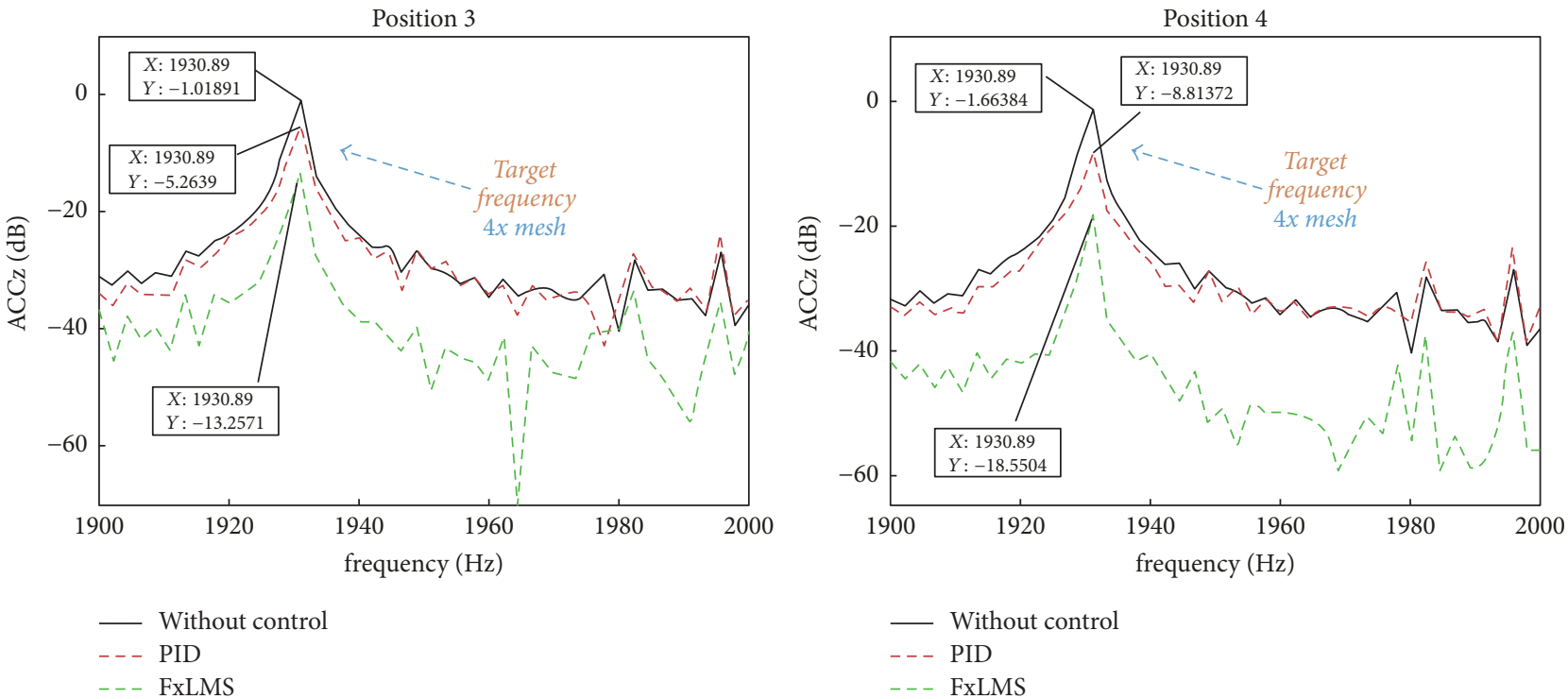

FIgURE 9: The cosimulation results of the four different positions for fixing the PZTs.

As shown in Figure 9, with respect to the four different positions for fixing the PZTs, both PID and FxLMS algorithms can suppress the $4 \mathrm{x}$ single target harmonic vibration to some extent. For position 1 , at the target frequency, about $3 \mathrm{~dB}$ vibration reduction can be obtained by PID control method, and about $5 \mathrm{~dB}$ vibration reduction can be dominated by FxLMS algorithm. For position 2, the PID control can suppress the vibration approximately $3 \mathrm{~dB}$, and FxLMS can suppress the vibration about $12.5 \mathrm{~dB}$. Similarly, for position 3 , the PID control can decrease the vibration about $4 \mathrm{~dB}$, and FxLMS algorithm can reduce the vibration up to $12 \mathrm{~dB}$. However, for position 4 , about $7 \mathrm{~dB}$ vibration can be reduced by $\mathrm{PID}$, and up to $17 \mathrm{~dB}$ vibration reduction can be controlled by FxLMS algorithm. Comparing the vibration reduction results of the four different positions, it can be inferred that the position which has larger vibration magnitude will exert a better control effect; in other words, position 4 is the better actuating position for locating the PZT. Although position division is not accurate enough, at least it can prove the correctness of the conclusion discussed by the modal analysis in Section 2 and provide an applicable method for finding an appropriate actuating position for PZT.

4.2. A Single Target Harmonic Vibration Control. In this part, the vibration control of a single gear mesh harmonic up to the fourth order for various rotational speeds was studied. We let the rotational speed $n=1524 \mathrm{r} / \mathrm{min}, 2032 \mathrm{r} / \mathrm{min}$, and $2540 \mathrm{r} / \mathrm{min}$ and load torque $T=1 \mathrm{~N} \cdot \mathrm{m}$ and $3 \mathrm{~N} \cdot \mathrm{m}$, respectively. In point of these situations, the vibration control simulations of MGTS based on PID and FxLMS algorithm are carried out to analyze the influences of the diversifications of rotational speed and load to the control effect. And the vibration control results of the input shaft at the input gear rotation center are given in Figure 10, and the control effects 

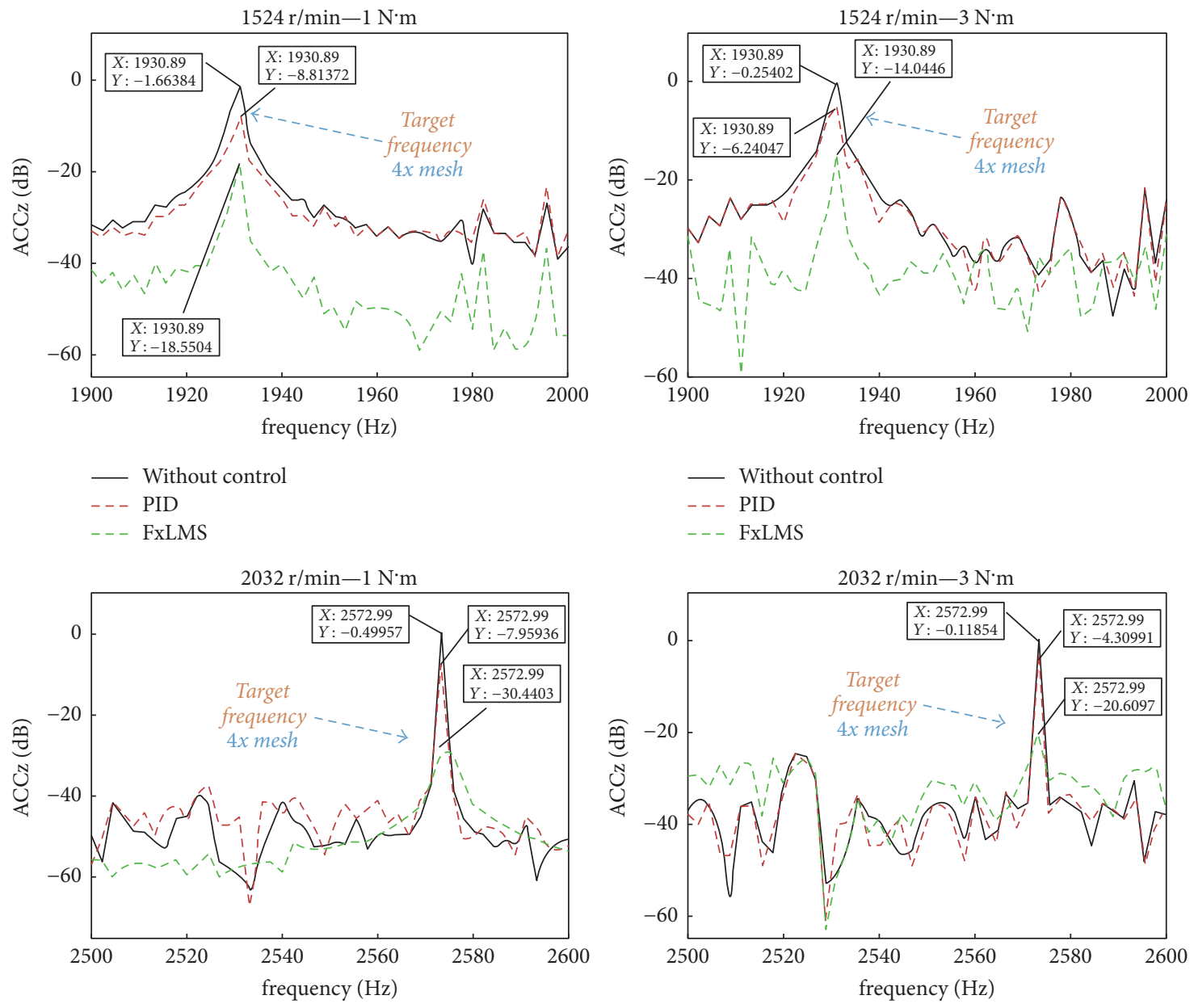

- Without control

- - PID

- - FxLMS

- Without control

- - PID

- _ FxLMS
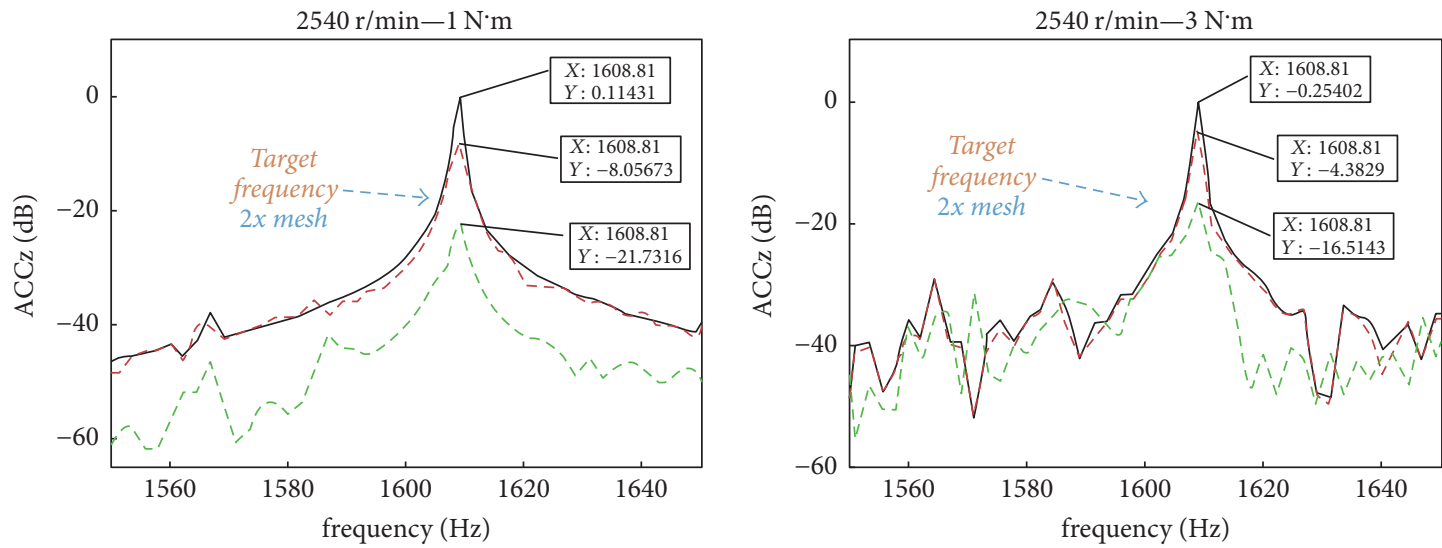

_ Without control
- - PID
- - FxLMS
— Without control$$
\text { - - - PID }
$$$$
\text { -. FxLMS }
$$

FIGURE 10: The vibration control results of the input shaft at the gear rotation center. 
TABLE 4: The comparison results of Figure 10.

\begin{tabular}{|c|c|c|c|c|c|c|}
\hline Condition $(\mathrm{r} / \mathrm{min} / 1 \mathrm{~N} \cdot \mathrm{m})$ & $1524 / 1$ & $1524 / 3$ & $2032 / 1$ & $2032 / 3$ & $2540 / 1$ & $2540 / 3$ \\
\hline Without control/(dB) & -1.66384 & -0.25402 & -0.49957 & -0.11854 & 0.11431 & -0.25402 \\
\hline $\mathrm{PID} /(\mathrm{dB})$ & -8.81372 & -6.24047 & -7.95936 & -4.30991 & -8.05673 & -4.3829 \\
\hline FxLMS/(dB) & -18.5504 & -14.0446 & -30.4403 & -20.6097 & -21.7316 & -16.5134 \\
\hline
\end{tabular}

TABLE 5: The comparison results of Figure 11.

\begin{tabular}{lcccccc}
\hline Condition $(\mathrm{r} / \mathrm{min} / \mathrm{l} \mathrm{N} \cdot \mathrm{m})$ & $1524 / 1$ & $1524 / 3$ & $2032 / 1$ & $2032 / 3$ & $2540 / 1$ & $-2540 / 3$ \\
\hline Without control/(dB) & -15.1778 & -1.8346 & -0.01693 & -0.71803 & -5.57155 \\
PID/(dB) & -24.6401 & -7.47954 & -8.8569 & -5.78154 & -14.8434 & -9.01214 \\
FxLMS/(dB) & -34.5487 & -17.3695 & -18.9738 & -10.845 & -28.5859 & -15.8312 \\
\hline
\end{tabular}

of the output shaft at the output gear rotation center are given in Figure 11.

According to Figures 10 and 11, it is obvious to know that, at the single target frequency, the control effect of FxLMS control algorithm is significantly better than PID control method. For instance, when the input shaft rotational speed $n=1524 \mathrm{r} / \mathrm{min}$ and the load torque $T=1 \mathrm{~N} \cdot \mathrm{m}$, with regard to the vibration control of input shaft at the gear rotation center, the vibration at $4 \mathrm{x}$ mesh target harmonic frequency can be suppressed up to $17 \mathrm{~dB}$ by the FxLMS control, and about $7 \mathrm{~dB}$ reduction can be obtained by $\mathrm{PID}$ control. In addition, for the vibration control of the output shaft at gear rotation center with the same conditions, the FxLMS control algorithm can suppress the vibration up to $19.3 \mathrm{~dB}$, and the PID control can cut down the vibration approximately to $7 \mathrm{~dB}$. As for the control effect of the other conditions, in order to analyze the control results conveniently, the comparisons of the results in Figures 10 and 11 are sorted out in Tables 4 and 5 .

By analyzing the above contents, for the various target harmonic vibrations at various conditions, it can be found that both PID control and FxLMS control methods can availably suppress the vibrations of the rotation shafts at the gear rotation center. But FxLMS control algorithm is more effective when facing the target harmonic frequency. Furthermore, under the circumstances of the input rotational speeds being the same, with the increase of the load torque, the general control effects of both control methods show a downward trend. Therefore, it has been proved that the load torque has a severe effect on the vibration control. And this result will have a guide for the future experimental study.

\section{Conclusions}

As a guide work for the future experimental study, a dynamic model with 26 DOFs was investigated using LPM in this paper. The modal analysis results solved by using the FEM were compared with the modal solutions resolved by the calculation method to validate the correctness of the dynamic modelling. Then a novel AVC structure of multistage gearbox with built-in PZTs was developed according to the modal results solved by FEM. In addition, in order to examine the feasibility and effectiveness of MGTS vibration control and ascertain the appropriate placement position of PZTs, a series of ADAMS and Matlab cosimulations were carried out based on the PID control and FxLMS adaptive control algorithm. On the basis of simulation results, we can draw several conclusions as follows. First, the compared results of the calculation method and FEM demonstrated the correctness of the modal solving. Second, it is feasible for determining a AVC structure of MGTS in the light of modal analysis. Third, the cosimulation results proved that PID and FxLMS adaptive control algorithms can suppress the gear mesh vibrations of the MGTS effectively. And FxLMS control algorithms are more robust and active than the classic PID control method. At last, the feasibility of the AVC method based on FxLMS algorithm for reducing the MGTS vibrations has been verified by cosimulations, and it will provide a considerable and forceful reference for the future experimental investigation.

\section{Nomenclature}

AVC: Active vibration control

CMM: Classical modelling methods

DEOM: Differential equations of motion

DOF: Degree of freedom

FEM: Finite element method

FGMF: Fundamental gear mesh frequency

FxLMS: Filtered-x least mean square

LMS: $\quad$ Least mean square

LPM: Lumped parameter method

MA: Modal analysis

MGTS: Multistage gear transmission system

MMM: Modern modelling methods

PID: $\quad$ Proportional Integral Derivate

PZT: Piezoelectric actuator.

\section{Conflicts of Interest}

The authors declare that there are no conflicts of interest regarding the publication of this paper.

\section{Acknowledgments}

This work is supported by National Natural Science Fund (no. 51405169). 

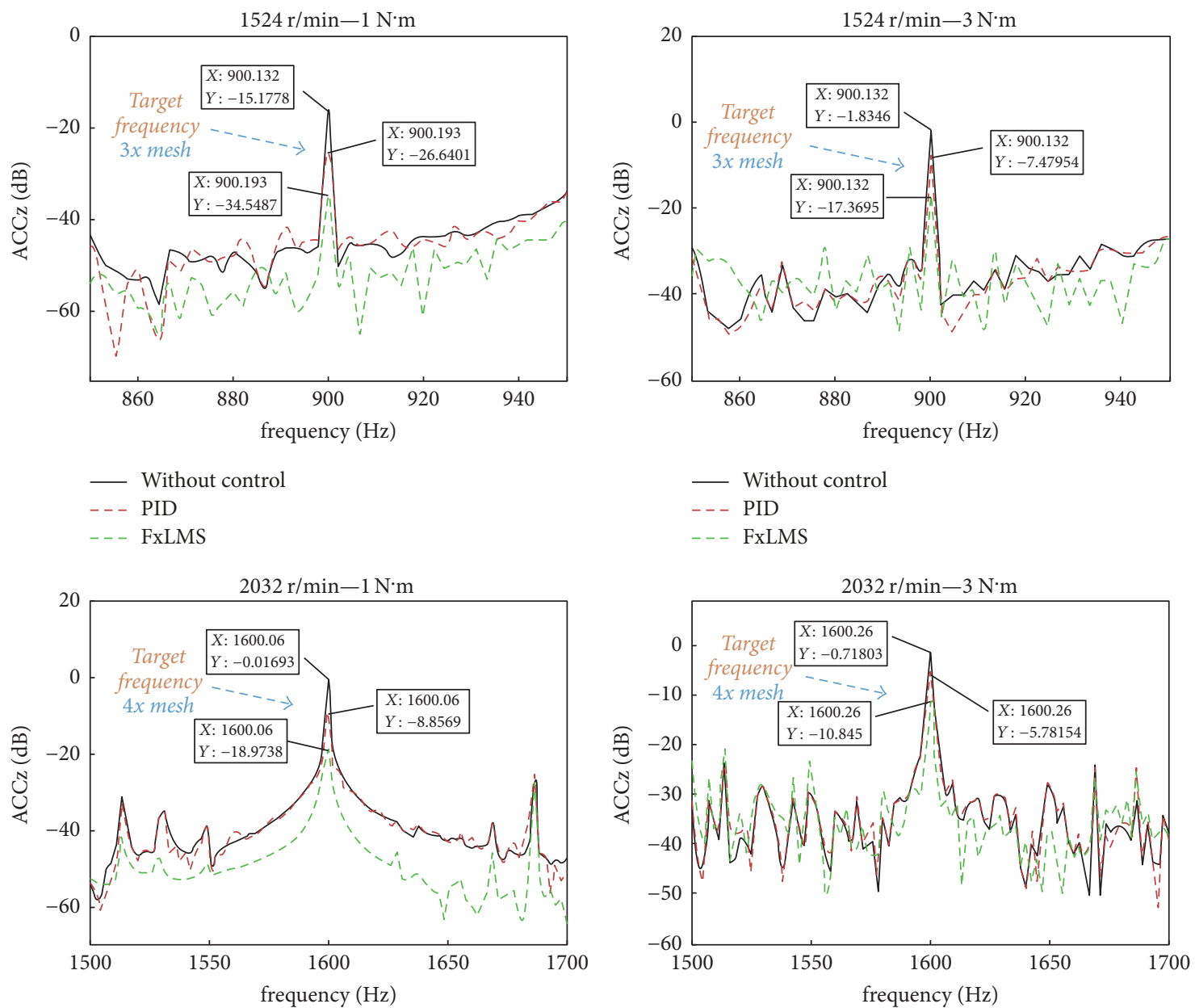

_ Without control
- - PID
- - FxLMS

— Without control

- - - PID

-. - FxLMS
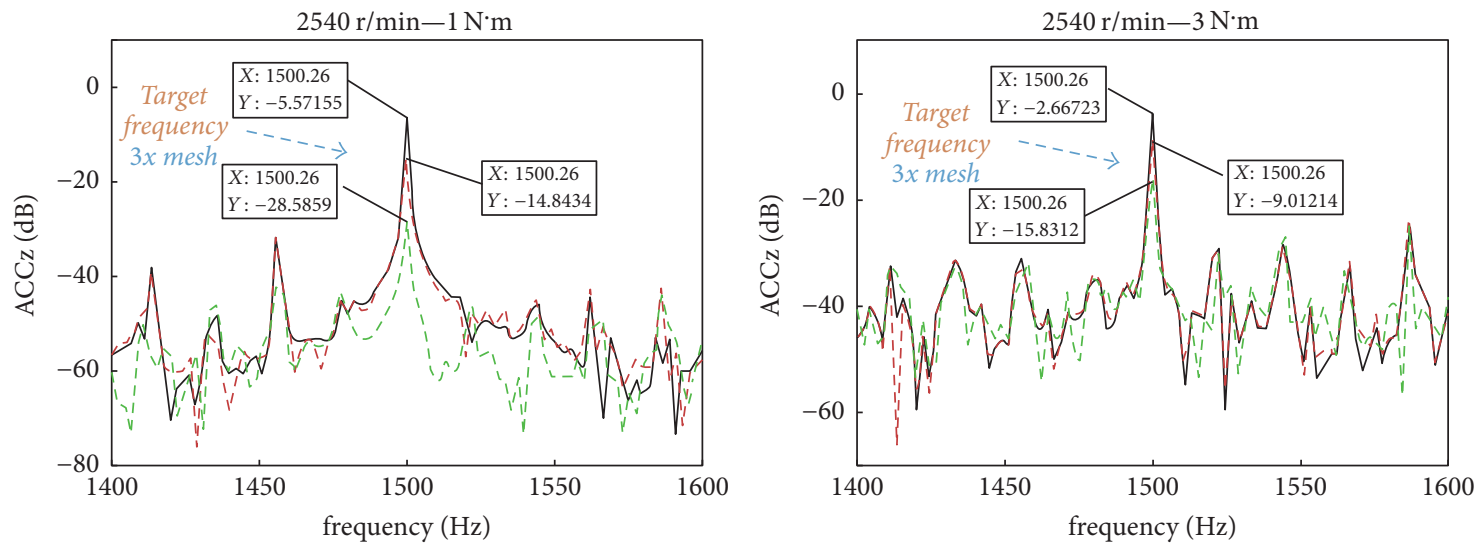

- Without control
-- PID

— Without control

- - FxLMS

- - - PID

-. - FxLMS

FIGURE 11: The vibration control results of the output shaft at the gear rotation center. 


\section{References}

[1] A. M. Simonović, M. M. Jovanović, N. S. Lukić, N. D. Zorić, S. N. Stupar, and S. S. Ilić, "Experimental studies on active vibration control of smart plate using a modified PID controller with optimal orientation of piezoelectric actuator," Journal of Vibration and Control, vol. 22, no. 11, pp. 2619-2631, 2014.

[2] C. U. Dogruer and A. K. Pirsoltan, "Active vibration control of a single-stage spur gearbox," Mechanical Systems and Signal Processing, vol. 85, pp. 429-444, 2017.

[3] G. T. Montague, D. Manchala, and E. Thomas, "Feedforward control of gear mesh vibration using piezoelectric actuators," Shock and Vibration, vol. 1, no. 5, pp. 473-484, 1994.

[4] B. Rebbechi, C. Howard, and C. Hansen, "Active control of gearbox vibration," in Proceedings of Active, pp. 295-304, 1999.

[5] M. H. Chen and M. J. Brennan, "Active control of gear vibration using specially configured sensors and actuators," Smart Materials and Structures, vol. 9, no. 3, pp. 342-350, 2000.

[6] Y. H. Guan, W. S. Shepard Jr., T. C. Lim, and M. Li, "Experimental analysis of an active vibration control system for gearboxes," Smart Materials and Structures, vol. 13, no. 5, pp. 1230-1237, 2004.

[7] M. Li, T. C. Lim, W. S. Shepard Jr., and Y. H. Guan, "Experimental active vibration control of gear mesh harmonics in a power recirculation gearbox system using a piezoelectric stack actuator," Smart Materials and Structures, vol. 14, no. 5, pp. 917927, 2005.

[8] M. Li, T. C. Lim, and W. S. Shepard Jr., "Modeling active vibration control of a geared rotor system," Smart Materials and Structures, vol. 13, no. 3, pp. 449-458, 2004.

[9] M. Li, T. C. Lim, Y. H. Guan, and W. S. Shepard Jr., "Actuator design and experimental validation for active gearbox vibration control," Smart Materials and Structures, vol. 15, no. 1, pp. N1N6, 2006.

[10] G. Pinte, S. Devos, B. Stallaert, W. Symens, J. Swevers, and P. Sas, "A piezo-based bearing for the active structural acoustic control of rotating machinery," Journal of Sound and Vibration, vol. 329, no. 9, pp. 1235-1253, 2010.

[11] Y. Pasco, O. Robin, P. Bélanger, A. Berry, and S. Rajan, "Multiinput multi-output feedforward control of multi-harmonic gearbox vibrations using parallel adaptive notch filters in the principal component space," Journal of Sound and Vibration, vol. 330, no. 22, pp. 5230-5244, 2011.

[12] P. Belanger, A. Berry, Y. Pasco, O. Robin, Y. St-Amant, and S. Rajan, "Multi-harmonic active structural acoustic control of a helicopter main transmission noise using the principal component analysis," Applied Acoustics, vol. 70, no. 1, pp. 153-164, 2009.

[13] W. Gao, L. Wang, and Y. Liu, "A modified adaptive filtering algorithm with online secondary path identification used for suppressing gearbox vibration," Journal of Mechanical Science and Technology, vol. 30, no. 11, pp. 4833-4843, 2016.

[14] G. Zhao, N. Alujević, B. Depraetere, G. Pinte, J. Swevers, and P. Sas, "Experimental study on active structural acoustic control of rotating machinery using rotating piezo-based inertial actuators," Journal of Sound and Vibration, vol. 348, pp. 15-30, 2015.

[15] X. Xu and R. Zhou, "Research on torsional vibration of gearshafting system based on an extended lumped parameter model," Advanced Materials Research, vol. 143-144, pp. 487-492, 2011.
[16] J. Zhang, Y. Song, and J. Xu, "A discrete lumped-parameter dynamic model for a planetary gear set with flexible ring gear," Applied Mechanics and Materials, vol. 86, pp. 756-761, 2011.

[17] T. M. Ericson and R. G. Parker, "Planetary gear modal vibration experiments and correlation against lumped-parameter and finite element models," Journal of Sound and Vibration, vol. 332, no. 9, pp. 2350-2375, 2013.

[18] A. Hammami, A. F. Del Rincon, F. V. Rueda, F. Chaari, and M. Haddar, "Modal analysis of back-to-back planetary gear: Experiments and correlation against lumped-parameter model," Journal of Theoretical and Applied Mechanics, vol. 53, no. 1, pp. 125-138, 2015.

[19] X. Tieming, Z. Shuiting, and Y. Liao, "Free modal analysis for spiral bevel gear wheel based on the lanczos method," Open Mechanical Engineering Journal, vol. 9, no. 1, pp. 637-645, 2015.

[20] S. Panda, B. K. Sahu, and P. K. Mohanty, "Design and performance analysis of PID controller for an automatic voltage regulator system using simplified particle swarm optimization," Journal of The Franklin Institute, vol. 349, no. 8, pp. 2609-2625, 2012.

[21] J.-L. Song, W.-L. Cheng, Z.-M. Xu, S. Yuan, and M.-H. Liu, "Study on PID temperature control performance of a novel PTC material with room temperature Curie point," International Journal of Heat and Mass Transfer, vol. 95, pp. 1038-1046, 2016.

[22] S. Chunsheng, J. Youliang, and Z. Jinguang, "PID Control of Multisources Complex Excitations Active Vibration Isolation System: An Improved Particle Swarm Optimization Algorithm," Shock and Vibration, vol. 2016, Article ID 3486959, 15 pages, 2016.

[23] M. Tahir Akhtar and W. Mitsuhashi, "Improving performance of FxLMS algorithm for active noise control of impulsive noise," Journal of Sound and Vibration, vol. 327, no. 3-5, pp. 647-656, 2009.

[24] Z. Qiu, C.-M. Lee, Z. H. Xu, and L. N. Sui, "A multi-resolution filtered-x LMS algorithm based on discrete wavelet transform for active noise control," Mechanical Systems and Signal Processing, vol. 66-67, pp. 458-469, 2016.

[25] L. Luo, J. Sun, and B. Huang, "A novel feedback active noise control for broadband chaotic noise and random noise," Applied Acoustics, vol. 116, pp. 229-237, 2017.

[26] Z. Bo, J. Yang, C. Sun, and S. Jiang, "A filtered-x weighted accumulated LMS algorithm: stochastic analysis and simulations for narrowband active noise control system," Signal Processing, vol. 104, pp. 296-310, 2014. 


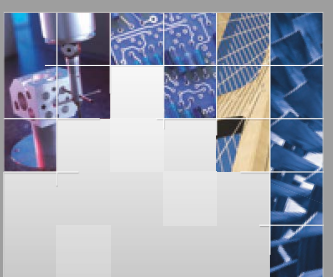

\section{Enfincering}
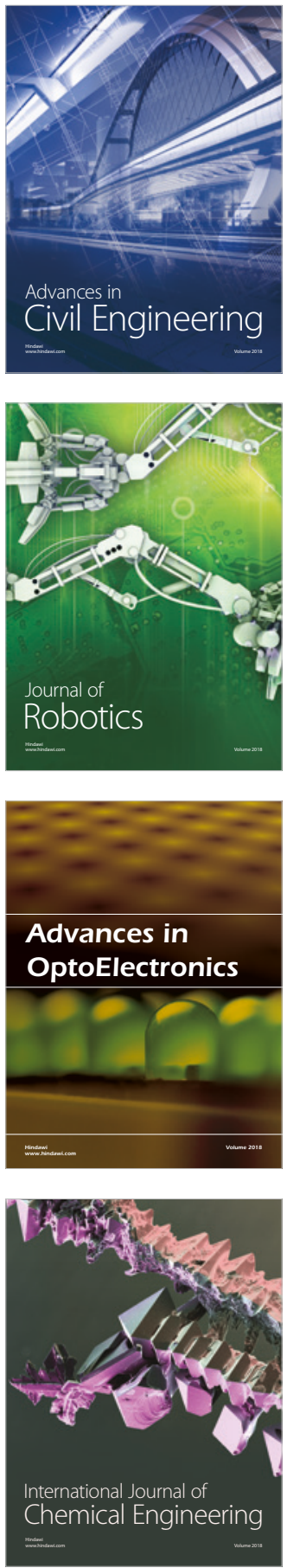

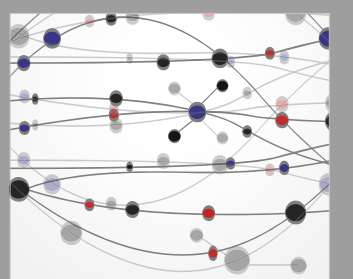

\section{Rotating \\ Machinery}

The Scientific World Journal

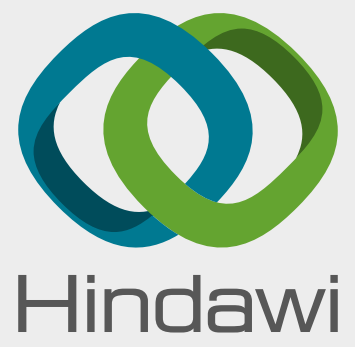

Submit your manuscripts at

www.hindawi.com
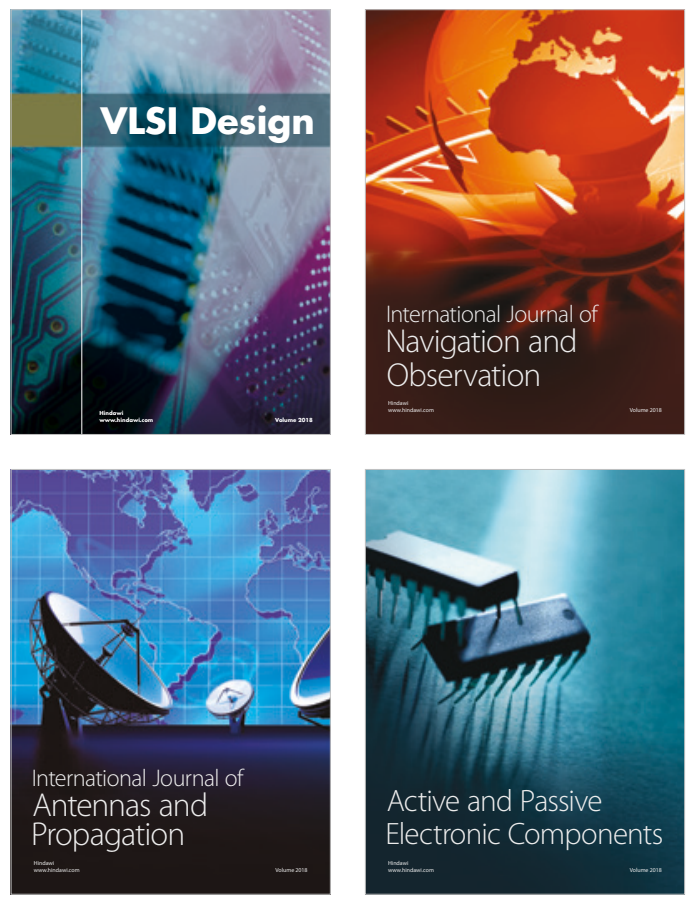
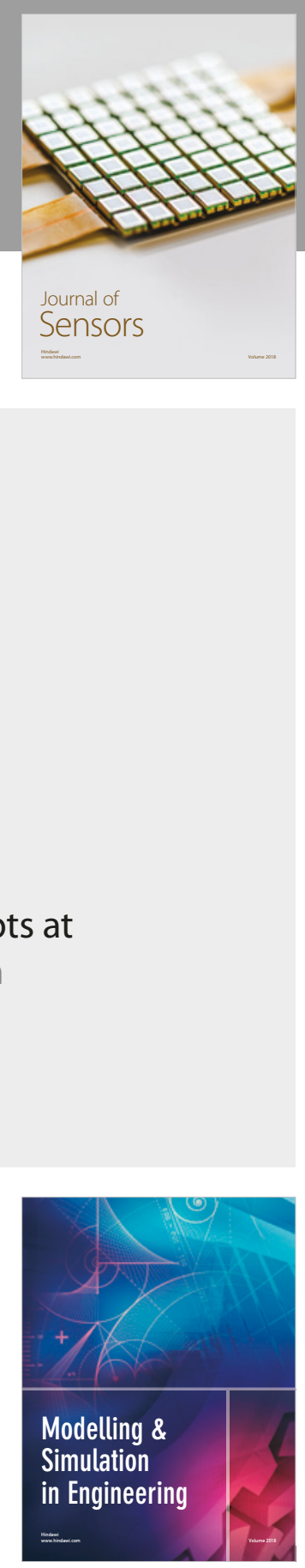

\section{Advances \\ Multimedia}
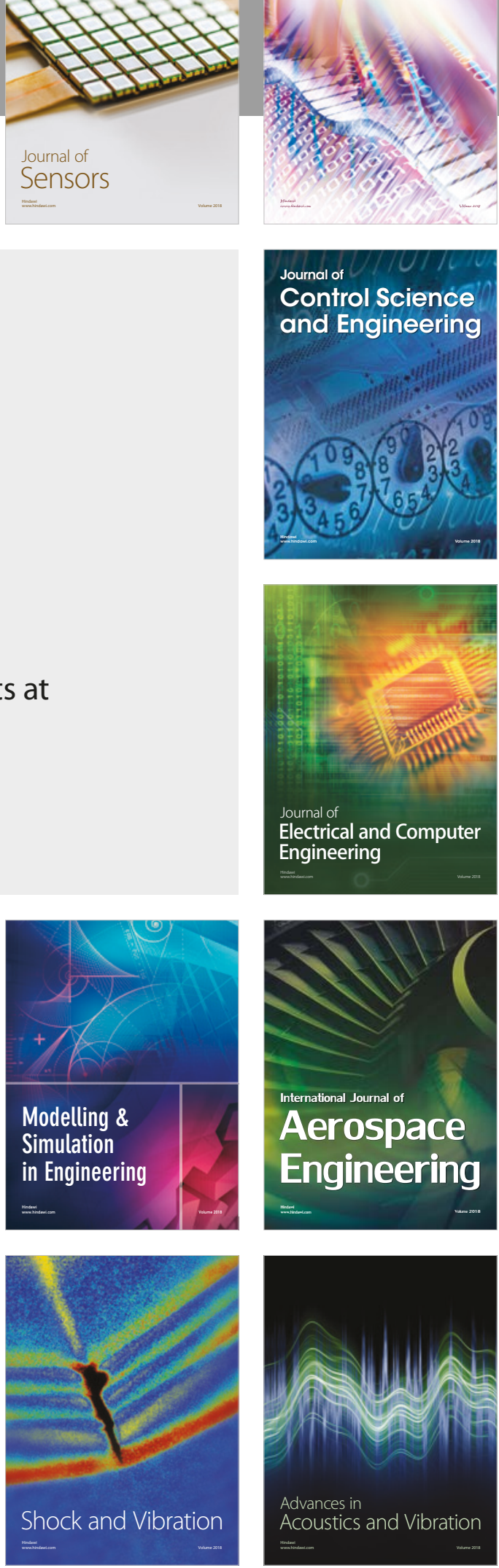